Estudios Públicos I6I (202I), 69-III

DOI: https://doi.org//0.38178/07/83089//653200629

Artículo

\title{
Impuestos a la renta de personas en Chile: simulaciones siguiendo esquemas de otros países OCDE
}

\author{
Adolfo Fuentes y Rodrigo Vergara \\ Centro de Estudios Públicos, Chile
}

Resumen: Es posible que Chile tenga que elevar a mediano plazo su carga tributaria para hacer frente a las mayores demandas sociales. La carga tributaria de Chile es alrededor de 3 puntos porcentuales del PIB inferior a la que tenían en promedio los países de la OCDE cuando alcanzaron nuestro PIB per cápita. El objetivo de este documento es explorar qué implica, en términos de recaudación y distribución del ingreso, el transitar hacia una estructura de impuesto al ingreso personal más parecida a la que tienen Australia, Nueva Zelanda, España y Noruega. Se concluye, en primer lugar, que de aplicarse la estructura tributaria de estos países a Chile, considerando el tipo de cambio nominal bilateral para los distintos tramos de ingreso, la mayor recaudación fluctuaría entre 1,5\% del PIB para la estructura de Australia hasta $4,7 \%$ del PIB en el caso de España. Si se ajustan las tablas de ingreso a

Adolfo Fuentes es Magíster en Economía, Universidad de Chile. Estudiante del programa de M.Res en Economía, University College London (UCL). Dirección: 30 Gordon Street, Kings Cross, Londres, Reino Unido. CP WC1H OAN. Email: adolfo.fuentes.20@ucl.ac.uk.

Rodrigo Vergara es PhD en Economía, Universidad de Harvard. Investigador senior del Centro de Estudios Públicos, Chile, e investigador asociado del Centro Mossavar-Rahmani de la Escuela de Gobierno (Kennedy), Universidad de Harvard. Dirección: Monseñor Sótero Sanz 162, Providencia, Santiago, Chile, CP 7500011 . Email: rvergara@cepchile.cl.

Agradecemos los comentarios de Nicolás Eyzaguirre, Carolina Fuensalida y Rodrigo Valdés, y la ayuda de Roberto Cases en la parte final de este documento, junto con los comentarios recibidos en un seminario interno del Centro de Estudios Públicos y de dos árbitros anónimos de Estudios Públicos. Adicionalmente, agradecemos la ayuda del Ministerio de DesarroIlo Social y Familia en las definiciones de ingresos utilizadas por la OCDE. 
tipo de cambio corregido por PPC, el rango va entre 3\% del PIB para la estructura de Australia hasta 5,2\% del PIB para la de Noruega. En segundo lugar, se concluye que la gran diferencia con Chile no son las tasas marginales máximas, sino que el tramo exento es relativamente elevado y, más importante, que las tasas marginales suben muy lentamente con el ingreso. Ello se traduce en que las tasas medias de impuesto en Chile son, en general, menores que en dichos países. Tercero, que con estas nuevas estructuras de impuesto, el mejoramiento en la distribución del ingreso es marginal. Sin embargo, si estos recursos extras se usan para aumentar las transferencias, se podría lograr una mejora más relevante.

PaLABRAS CLAVE: impuestos, ingreso personal, OCDE, transferencias, tasas de impuesto al ingreso personal

RECIBIDO: junio 2020 / ACEPTADO: noviembre 2020

\section{Personal Income Tax in Chile: Simulations with Other Countries' Structure}

ABSTRACT: It is likely that in the medium-term Chile will have to increase tax revenues as share of GDP to meet increasing demand for social services. Chile's tax revenue is 3 percentage points of GDP lower that the average of OECD countries when these countries had a per capita income similar to the one of today's Chile. The purpose of this article is to investigate what implies in terms of tax revenues and income distribution if Chile moves towards a personal income tax structure similar to the one that today have Australia, New Zealand, Spain and Norway. We conclude: firstly, if we use the nominal exchange rate for the different income brackets, there would be an increase in the personal income tax revenue of between 1.5\% of GDP for the Australian tax structure and $4.7 \%$ of GDP for the case of Spain. If we adjust the income brackets by purchasing power parity the range goes from $3 \%$ of GDP for the structure of Australia to $5.2 \%$ of GDP for the case of Norway. Secondly, this gap is explained not by the marginal income tax rate, but by the fact the exempt bracket is relatively high in Chile and, most importantly, tax rates increase too gradually in Chile as compared to these other four countries, hence making average tax rates in general lower. Thirdly, with this new tax structures income distribution improves only marginally. However, if these extra resources are used to make social transfers a significant improvement in income distribution can be achieved.

KEYwords: taxes, personal income, OECD, income transfers, personal income tax rates

ReCeIved: June 2020 / AcCEPTED: November 2020 


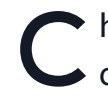
hile posee al menos dos particularidades en su estructura tributaria que lo destacan del resto de los países de la OCDE (Fuentes y Vergara 2020). En primer lugar, y como muestra el Gráfico 1, si comparamos a Chile con los países de la OCDE cuando tenían nuestro ingreso per cápita de 2018, veremos que Chile recauda 4,7 puntos del PIB por concepto de impuestos a las empresas, mientras que la mediana y promedio de los países OCDE es de 2,6 y 2,7 puntos del PIB, respectivamente. Aunque estas mediciones no están exentas de problemas de medición, ${ }^{1}$ esto sugiere que la carga tributaria de las empresas en Chile es relativamente elevada. Más aún, en las últimas dos décadas, mientras la tasa de impuesto corporativo en Chile ha subido, en los otros países, en promedio, ha bajado. Como se puede observar en el Gráfico 2, la mediana de la tasa de impuesto corporativo ${ }^{2}$ en la OCDE en 2000 era 32,5\%, mientras que en 2018 había bajado a 23\%. En Chile, en tanto, en igual período subió de $15 \%$ a $27 \%$.

Por otro lado, la recaudación por concepto de impuestos a la renta de las personas es particularmente baja. ${ }^{3}$ Como muestra el Gráfico 3, Chile recauda 1,4 puntos del PIB por este concepto, mientras que la me-

\footnotetext{
${ }^{1}$ La OCDE define la recaudación por impuestos corporativos como aquella tributación que es carga a sociedades o instituciones, indiferentemente de la situación personal de los dueños. Esto se debe a que los países tienen distintas formas de relacionar la tributación personal y aquella de sociedades, y realizar un ejercicio para separarlas no es evidente. En este sentido, es importante tener en consideración que los datos expresados no son influenciados por los créditos tributarios que se pueden obtener de la tributación de las empresas por parte de sus dueños (como ocurre con el caso de Chile con el impuesto de primera categoría y el global complementario). En este sentido, las series presentadas aquí son comparables entre países. Sin embargo, hay un aspecto en el cual dicha comparación sufre de problemas. Esto es en cuanto hay países en que el sistema tributario permite que, generalmente en empresas pequeñas y de pocos dueños, las utilidades se atribuyan directamente a los dueños que así pagan el impuesto personal. En los datos de la OCDE, por lo dicho previamente, esto es parte de las estadísticas del impuesto al ingreso personal. Ello hace aumentar la recaudación medida por este impuesto en desmedro de la recaudación medida por el impuesto corporativo. Esto podría explicar parte de la diferencia entre Chile y la media de los países OCDE en cuanto a la carga de ambos tipos de impuestos. Desgraciadamente, no tenemos la información desagregada como para hacer la comparación correcta.

${ }^{2}$ Con esta tasa nos referimos a la tasa combinada que incluye las tasas locales.

${ }^{3} \mathrm{Al}$ igual que en el caso de las empresas, este impuesto mide la recaudación por concepto de impuestos cargados a las personas, considerando sus beneficios tributarios asociados de participaciones en sociedades. Por lo mismo señalado antes, no es necesariamente comparable entre todos los países, debido a la existencia de empresas individuales o de pocos socios que tributan directamente a nivel del ingreso personal, por lo que en las estadísticas son consideradas carga de personas, no de empresas.
} 
Gráfico 1. RECAUDACIÓN TRIBUTARIA POR IMPUESTOS CARGADOS A LAS EMPRESAS

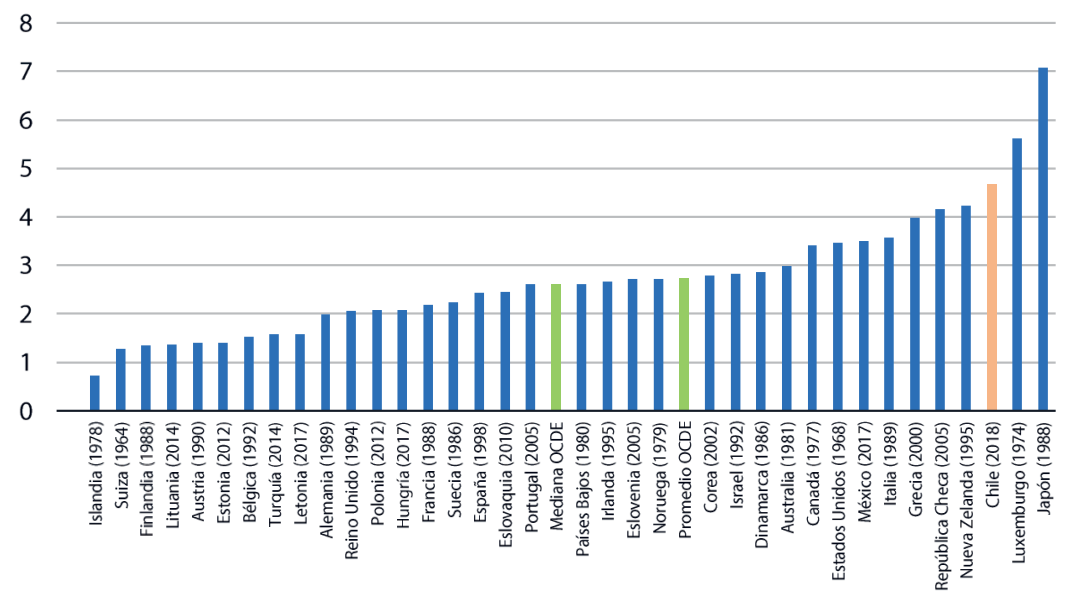

Fuente: Elaboración propia en base a datos de la OCDE.

Gráfico 2. EVOLUCIÓN TASA DE IMPUESTO CORPORATIVO (EN PORCENTAJE) EN CHILE Y EN LA OCDE

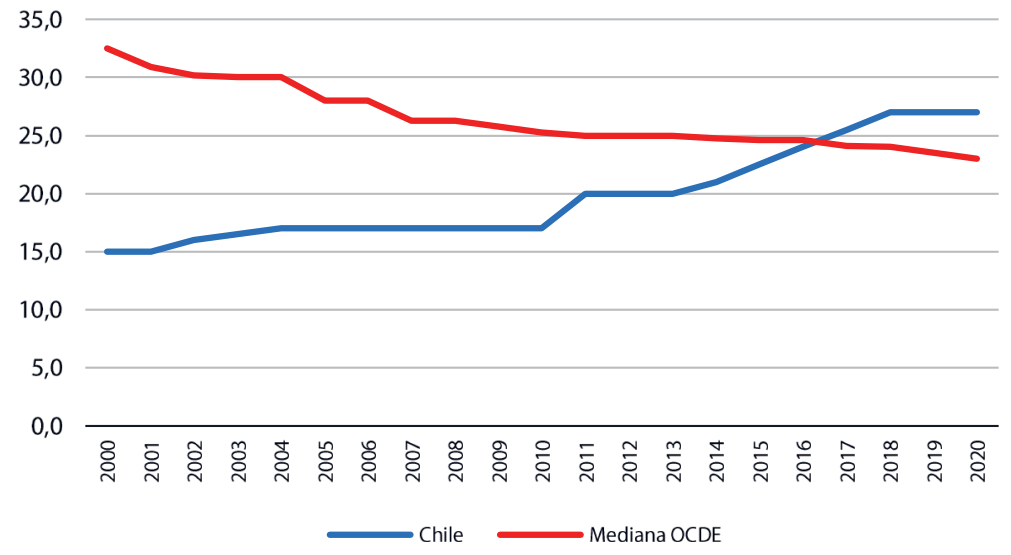

Fuente: Elaboración propia en base a datos de la OCDE.

diana y el promedio de los países OCDE asciende a 6,8 y 8,1 puntos del $\mathrm{PIB}$, respectivamente. En materia de impuesto a bienes y servicios, Chile recauda una cifra similar a la de los países de la OCDE cuando tenían un PIB per cápita similar al nuestro en la actualidad (Fuentes y Vergara 2020). 


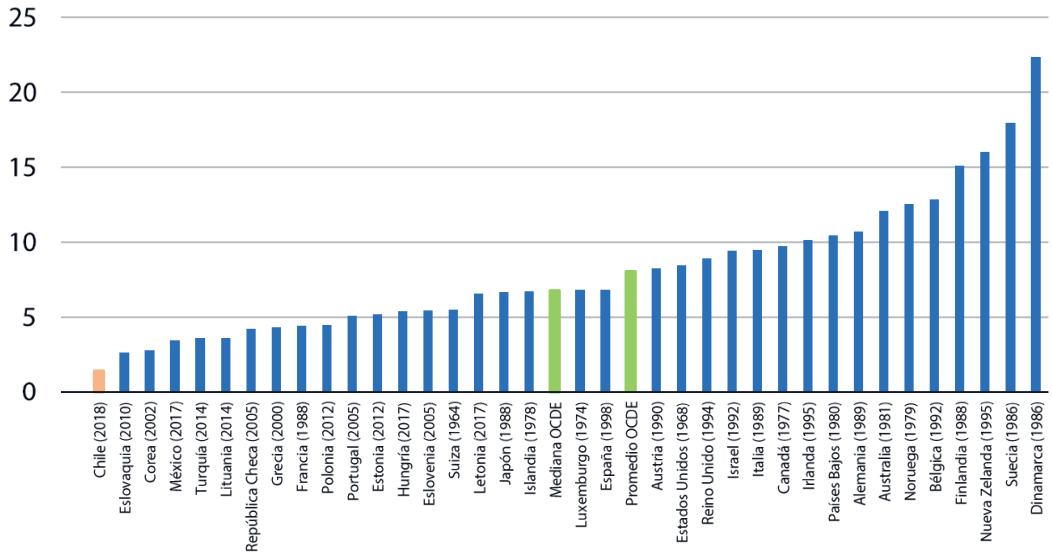

Fuente: Elaboración propia en base a datos de la OCDE.

Gráfico 4. DISTINTOS COEFICIENTES DE GINI PARA LOS PAÍSES DE LA OCDE

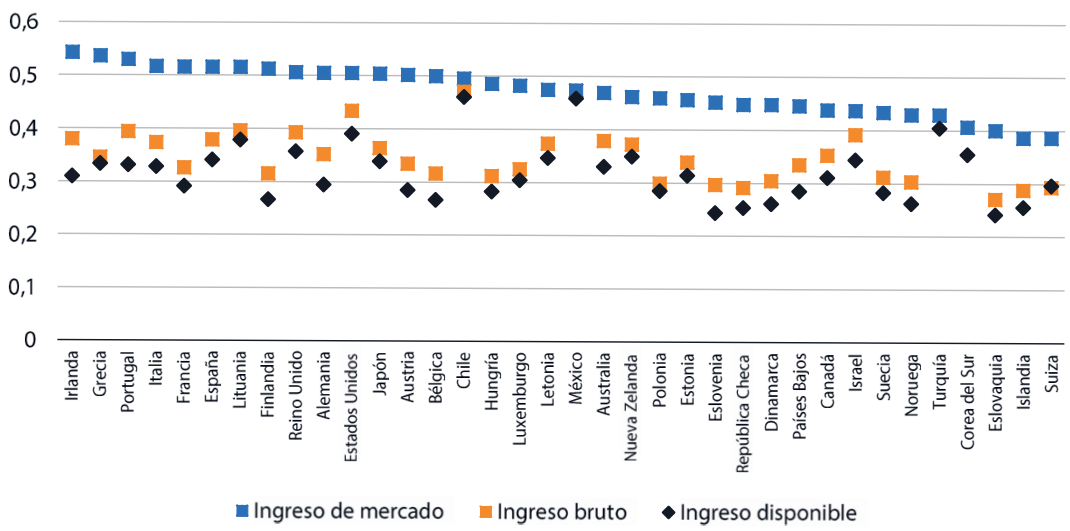

Fuente: Elaboración propia en base a datos de la OCDE (extraídos directamente, sin ser trabajados ni procesados).

Adicionalmente, es conocido que cuando se consideran los ingresos de mercado 4 de las personas, Chile no es particularmente desigual. En

\footnotetext{
${ }^{4}$ En inglés market income. Los ingresos de mercado corresponden a estimaciones de los ingresos que reciben los trabajadores, siguiendo una metodología común de la OCDE, a modo de que sean comparables entre los distintos países. Estos ingresos comprenden los ingresos brutos del empleo asalariado y del empleo cuenta propia. Adicionalmente se consideran los ingresos del capital, las pensiones recibidas del sistema de AFP, y el neto de las transferencias recibidas y pagadas desde/a otros hogares y organizaciones sin fines de lucro.
} 
efecto, como se aprecia en el Gráfico 4, está cerca de la mediana de los países de la OCDE. No obstante, cuando se consideran los ingresos brutos $^{5}$ y los ingresos disponibles, ${ }^{6}$ la situación cambia, en cuanto en dichos países mejora sustantivamente dicha distribución, mientras que en Chile no. En otras palabras, nuestro sistema tributario y de transferencias no hacen la gran diferencia que sí hacen en otras latitudes. Es importante destacar, como se aprecia en el mismo Gráfico 4, que la mayor diferencia en dichos países en términos de desigualdad la hacen las transferencias más que los impuestos, esto es, la distancia entre los ingresos de mercado y los ingresos brutos.

Por otra parte, es posible que Chile tenga que elevar a mediano plazo su carga tributaria para hacer frente a las mayores demandas sociales. Hoy nuestra carga es alrededor de 3 puntos porcentuales del PIB inferior a la que tenían en promedio los países de la OCDE cuando alcanzaron nuestro PIB per cápita (Fuentes y Vergara 2020). Ello mismo hace que dicha cifra se pueda usar como referencia. Ahora bien, si se decide aumentar la carga tributaria general, por una parte, y, por otra, la carga del impuesto al ingreso de las personas es baja, lo natural sería revisar nuestro sistema de impuesto al ingreso personal y compararlo con el que tienen otros países. Así, por ejemplo, si se recaudara lo mismo que la mediana de la OCDE con este impuesto, se obtendrían 5,4 puntos del PIB adicionales, o bien, cerca de US\$16 mil millones. ${ }^{7}$ Una fracción de ello sería suficiente para llevar a cabo la agenda social y a su vez recortar la carga tributaria de las empresas, que es demasiado elevada en términos comparados y nos hace menos competitivos para la inversión (e.g. Vartia 2008; Djankov et al. 2010).

\footnotetext{
${ }^{5}$ En inglés gross income. Los ingresos brutos corresponden al ingreso de mercado más las transferencias recibidas desde los esquemas de seguridad social y menos los pagos generados a estos sistemas asociados al empleo. En el caso de Chile, las transferencias recibidas consideran, entre otros, el pago del seguro de cesantía, el pago de pensiones en el sistema antiguo, y los aportes del INP, la PBS, el APS, las asignaciones familiares y bonos. Por su parte, los pagos consideran, entre otros, los pagos de cotizaciones a las AFP y los pagos del seguro de salud a Fonasa o Isapre.

${ }^{6}$ En inglés disposable income. El ingreso disponible corresponde al ingreso bruto menos los pagos de impuestos a la renta y la riqueza, que en el caso de Chile corresponden a una estimación del pago del Impuesto Global Complementario y/o Segunda Categoría.

${ }^{7}$ Considerando el PIB a precios corrientes de Chile, en 2018, de US\$298 mil millones. Fuente: Banco Mundial. Disponible en: https://datos.bancomundial.org/indicador/NY.GDP.MKTP. CD [20 de julio 2020].
} 
El objetivo de este artículo es explorar qué implica, en términos de recaudación y distribución del ingreso, el transitar hacia una estructura de impuesto al ingreso personal más parecida a la que tienen algunos países de la OCDE. Para ello tomamos cuatro países como referencia: Australia, Nueva Zelanda, España y Noruega. Los dos primeros son países exportadores de materias primas y muchos han planteado la idea de seguir sus caminos de desarrollo (ver, por ejemplo, Briones 2020). Tomamos a España por nuestra ascendencia cultural y porque es un país que en general ha tomado una senda socialdemócrata. Noruega, por su parte, es un país nórdico, caracterizado por una alta participación del Estado, masivos programas sociales, pero además dependiente de un recurso natural (el petróleo).

El ejercicio que hacemos es el siguiente: cuánto adicional se recaudaría en Chile si se tuviera una estructura de impuesto al ingreso de las personas similar al de estos países. Analizamos también cuánto cambiaría la distribución del ingreso (medida por el coeficiente Gini) con este cambio tributario y, luego, cuánto más podría mejorar dicha distribución si los recursos generados fueran en algún porcentaje a mayores transferencias.

Para asegurarnos de que se están haciendo las comparaciones correctas, lo primero que se debe hacer es, a partir de nuestra fuente primaria de información sobre los ingresos de la población (la encuesta Casen del año 2017), ${ }^{8}$ intentar replicar, a partir del sistema tributario chileno, su recaudación. Una vez que tenemos una buena caracterización de los ingresos tributarios de las personas en Chile y que los agregados de recaudación coinciden con los efectivos reportados por el Servicio de Impuestos Internos (SII), se aplican las estructuras tributarias de esos cuatro países a Chile. El ejercicio no es simple, porque las estructuras tributarias de los países no lo son. Cada país cuenta con distintas exenciones y casos particulares, los que implican reducciones de la renta imponible o créditos tributarios, o consideran distintas tasas según la localidad, entre otros. Aunque estimamos que logramos capturar algunas de esas complejidades, de seguro no las captamos todas. En particular, el ejercicio

\footnotetext{
${ }^{8}$ La encuesta de caracterización socioeconómica nacional (Casen) es una encuesta con representatividad nacional y regional a nivel de hogares encargada cada dos o tres años por el Ministerio de Desarrollo Social y Familia (MDSF) a distintas organizaciones académicas nacionales desde 1990. Su objetivo principal es generar una medición de la pobreza de ingresos y, desde 2015, de la pobreza multidimensional. Es la principal encuesta utilizada en Chile para medir distribución del ingreso.
} 
busca capturar la estructura del impuesto al ingreso (definido como sus tasas y tramos), pero no sus distintas excepciones. En efecto, se toma el sistema chileno y se le aplican los tramos, tasas, créditos y deducibles de otros países. Por la complejidad de las legislaciones tributarias, este ejercicio tiene que ser considerado como una aproximación a la realidad. Lo importante es que las magnitudes que encontramos no son menores y ello confirma que hay diferencias importantes, más allá del número exacto de dicha diferencia.

Nuestros principales resultados son los siguientes. Primero, de aplicarse la estructura tributaria de estos países a Chile y considerando el tipo de cambio nominal bilateral para los distintos tramos de ingreso (es decir, los ingresos en su moneda multiplicado por el tipo de cambio peso/moneda del país), la mayor recaudación fluctuaría entre 1,5\% del PIB para la estructura de Australia hasta 4,7\% del PIB en el caso de España $(3,6 \%$ y $4,4 \%$ del PIB para Nueva Zelanda y Noruega, respectivamente). Si se ajustan las tablas de ingreso al tipo de cambio corregido por $\mathrm{PPC}^{9}{ }^{9}$ el rango va entre $3 \%$ del PIB para la estructura de Australia hasta $5,2 \%$ del PIB para la de Noruega ( $4,3 \%$ y $4,9 \%$ del PIB para Nueva Zelanda y España, respectivamente). Segundo, la gran diferencia no son las tasas marginales máximas, que no son muy diferentes a la chilena, sino toda la estructura de tasas y, en particular, su pendiente. Así, en estos países se empieza a pagar impuesto a la renta con ingresos relativamente bajos y las tasas de impuesto suben mucho más rápido que en Chile. Por ejemplo, si consideramos las escalas a tipo de cambio nominal, una persona que en Chile tenía un ingreso imponible de 2 millones de pesos al mes pagaba una tasa media de 3,9\% (en 2017). Sin embargo, con ese mismo ingreso, si se usara la escala de impuestos de esos países, pagaría entre $13,2 \%$ y $18,9 \%{ }^{10}$ Tercero, con estas nuevas estructuras de impuesto, y asumiendo que el gasto de la recaudación extra se gasta en ítems que no afectan la distribución del ingreso, el coeficiente de Gini de los ingresos monetarios bajaría desde el 0,49 actual a un rango entre 0,46 y 0,48, dependiendo de qué país se tome como estructura y si es en términos nominales o a PPC. Esto es, no hay un cambio muy significativo en la distribución del ingreso. Sin embargo, si esos recursos se usan en transfe-

\footnotetext{
${ }^{9}$ Paridad de poder de compra. En inglés Pushasing Parity Power (PPP).

10 Si los tramos de impuestos se ajustaran a PPC, entonces el rango sería entre 19,4\% y 22,6\%. Los cálculos se hacen para 2017, dado que ese es el año de la última Casen.
} 
rencias focalizadas, la baja en el Gini sería mayor. ${ }^{11}$ Se hacen ejercicios suponiendo que las transferencias aumentan en distintos porcentajes de la recaudación, y ello produce mejoras más significativas en el coeficiente Gini. En otras palabras, la mejora en la recaudación, tal como se mencionó previamente para la gran mayoría de los países, se produce más por las transferencias que se pueden financiar con los recursos adicionales que por los mayores impuestos propiamente tales.

Este artículo se organiza de la siguiente forma. En la sección 1 se explicita la metodología a usar y las fuentes de datos. Asimismo, se hace el ejercicio de calcular la recaudación del impuesto a la renta personal en Chile con la estructura que tiene este impuesto y la distribución que obtenemos de la Casen. Ello, para cerciorarnos de que los ejercicios a continuación se hacen a partir de una base sólida. La sección 2 calcula la recaudación adicional que se obtendría en Chile con las estructuras de impuesto a la renta de Australia, España, Noruega y Nueva Zelanda. Se hacen dos ejercicios por país. En el primero se aplica la estructura tributaria de esos países a Chile, considerando el tipo de cambio nominal. Esto es, se toman los distintos tramos del impuesto y los límites se llevan a pesos chilenos a través del tipo de cambio nominal. En el segundo, los ingresos se ajustan por PPC. Como para estos casos en Chile una unidad de la moneda extranjera compra más que en los distintos países utilizados, resultará que los tramos considerados por el tipo de cambio ajustado por PPC serán menores a los considerados por el tipo de cambio nominal ( $y$, luego, la recaudación será mayor). Finalmente, la sección 3 presenta las conclusiones.

\section{Metodología y escenario base}

Para este ejercicio utilizaremos la encuesta Casen del último año disponible, esto es 2017. Esta encuesta contiene datos socioeconómicos de 70.948 hogares chilenos, los cuales cuentan con representación nacional y regional. Dentro de los ingresos de las personas, se detallan los ingresos autónomos y subsidios monetarios que reciben, de forma que podemos llevar a cabo nuestras simulaciones.

\footnotetext{
${ }^{11}$ Recordemos que en Chile los conceptos de ingreso más utilizados para medir la distribución del ingreso son el 'ingreso autónomo' (ingresos propios menos impuestos al ingreso personal) e 'ingreso monetario' (ingreso autónomo más transferencias). Mayor detalle se encuentra más adelante.
} 
Antes de comenzar a detallar la metodología, es importante mencionar algunos aspectos que limitan nuestros resultados. En primer lugar, se debe tener claridad de que los datos Casen son autorreportados, vale decir, las personas pueden no ser precisas con respecto a sus ingresos y transferencias. Además, existe una subestimación de ingresos para el 1\% de mayores ingresos, lo que distorsiona en una medida relevante cálculos de distribución de ingreso (López, Figueroa y Gutiérrez 2013; Larrañaga y Rodríguez 2014; Fairfield y Jorrat 2014; López, Figueroa y Gutiérrez 2016). En segundo lugar, este ejercicio utiliza varios supuestos, de forma que sus resultados son sensibles a ellos. ${ }^{12} \mathrm{Si}$ bien creemos que nuestros supuestos son razonables, este ejercicio pretende proveer de órdenes de magnitud y no de resultados exactos. Por último, dado que estamos utilizando una encuesta cuyos datos se levantaron a fines de 2017 e inicios de 2018, es evidente que sus datos no reflejan la situación socioeconómica actual de los hogares chilenos, la cual puede haber cambiado después de los grandes hitos que hemos vivido en el último tiempo, como el estallido social o la crisis sanitaria provocada por el Covid-19.

En cuanto a la metodología, un primer desafío corresponde al período temporal al que asignaremos los ingresos de las personas. La encuesta Casen reporta que los hogares fueron entrevistados entre noviembre de 2017 y febrero de $2018 .{ }^{13}$ La distribución de los hogares entre estos meses es la que indica la Tabla 1 a continuación:

Tabla 1. DISTRIBUCIÓN DE ENCUESTAS A HOGARES SEGÚN MES DE LA ENCUESTA

\begin{tabular}{lcc}
\hline Mes & Cantidad de hogares & Porcentaje del total (\%) \\
\hline nov-17 & 9.406 & 13,3 \\
dic-17 & 26.394 & 37,2 \\
ene-18 & 32.337 & 45,6 \\
feb-18 & 2.811 & 4,0 \\
Total & 70.948 & 100,0 \\
\hline
\end{tabular}

Fuente: Elaboración propia sobre la base de datos de la encuesta Casen (2017).

Dado que el $96 \%$ de la muestra está considerada entre noviembre de 2017 y enero de 2018, asumiremos para todos los efectos de este

\footnotetext{
${ }^{12}$ Los supuestos serán explicitados en la medida en que son utilizados.

${ }^{13}$ Esto está documentado en la base de datos de la encuesta en el ítem llamado fecha_mes.
} 
ejercicio que los ingresos declarados corresponden al mes de diciembre de 2017, y que estos son representativos del resto del año 2017. Este supuesto simplifica significativamente el análisis a realizar, ya que al momento de calcular los impuestos mensuales solo consideraremos la tabla de un mes y no las cuatro tablas posibles. En todo caso, utilizar la tabla correspondiente a cada mes no cambia mayormente nuestros cálculos ya que, con inflaciones bajas, las tablas mensuales varían poco de mes a mes. Adicionalmente, asumir que los ingresos de un período son representativos del resto del año es un supuesto bastante común en la literatura que estudia desigualdad de ingresos y, en especial, en los cálculos del coeficiente de Gini (Contreras 1999; Larrañaga y Herrera 2008; Rau 2011; Beyer 2011, entre otros).

Definida la temporalidad de los ingresos, el desafío siguiente corresponde a que el sistema tributario chileno cuenta con impuestos que las personas pagan mensualmente y que grava los ingresos del trabajo (el impuesto de segunda categoría, ISC) e impuestos que se pagan anualmente y gravan las rentas del trabajo y las rentas del capital (el impuesto global complementario, IGC). Dada la naturaleza del IGC, el pago de ISC actúa como crédito, de forma que para poder determinar el pago total de impuestos se hace necesario calcular primero el ISC y, posteriormente, el IGC.

Para ello, clasificaremos los ingresos de las personas siguiendo la clasificación Casen que está reflejada en el Gráfico 5.

Los ingresos monetarios de las personas corresponden a la suma de los ingresos autónomos - aquellos que generan por sí mismos-y de las transferencias monetarias - aquellos ingresos que les son transferidos desde el Estado. Los ingresos autónomos se pueden dividir a su vez en dos grupos: los ingresos del trabajo -aquellos que se generan por trabajar de forma dependiente o independiente- y otros ingresos que son aquellos ingresos autónomos que no provienen del trabajo como ganancias de capital, arriendos, dividendos de acciones, entre otros.

Para calcular el ISC debemos considerar que las rentas del trabajo declaradas en la encuesta Casen son montos líquidos. Por lo tanto, para obtener los ingresos imponibles debemos clasificar a los distintos trabajadores en grupos según sus condiciones laborales, de forma de entender los ajustes que debemos hacer sobre sus ingresos líquidos para estimar sus ingresos imponibles. 
Gráfico 5. DISTINTOS TIPOS DE INGRESOS CONSIDERADOS

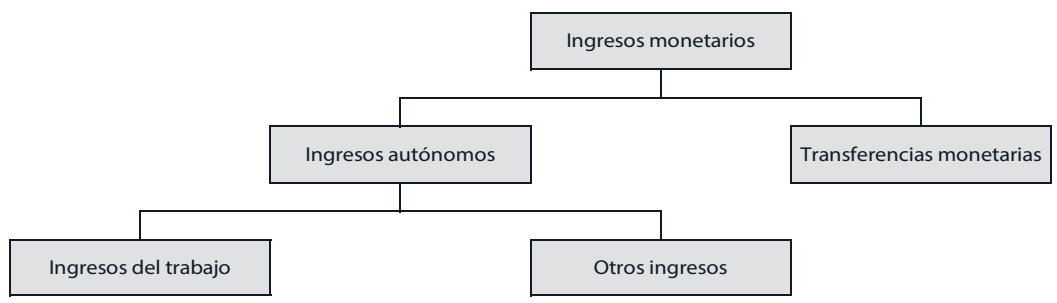

Fuente: Elaboración propia.

Las categorías que utilizaremos para dividir a los trabajadores serán las siguientes:

- $\quad$ Si entrega boleta por su trabajo en su ocupación principal.

- Si realiza la cotización obligatoria como trabajador dependiente en su ocupación principal.

- $\quad$ Si tiene contrato de trabajo en su ocupación principal.

En primer lugar, dividiremos a los trabajadores entre aquellos que entregan boleta y aquellos que no lo hacen. Si el trabajador entrega boleta diremos que pertenece al grupo 1 y su base tributable corresponde a:

Base tributable $_{1}=\frac{\text { Monto líquido }_{1}}{0,9}$

La retención del $10 \%{ }^{14}$ es considerada recaudación del ISC por parte del SII, por lo que también lo consideraremos de esa forma para poder respaldar nuestros resultados con las cifras entregadas por este servicio.

A continuación, a aquellos trabajadores que no entreguen boleta, los dividiremos en dos grupos: los que realizan la cotización obligatoria de trabajadores dependientes y aquellos que no lo hacen. Dentro de la cotización de trabajadores dependientes se incluye no solo a aquellos que cotizan en el sistema de AFP, sino también a aquellos que cotizan

\footnotetext{
${ }^{14}$ Esta retención subió a 10,75\% en 2020. Como nuestros cálculos son para 2017, este cambio no nos afecta. La serie se puede consultar en http://www.sii.cl/sobre_el_sii/serie_de_ingresos_tributarios.html.
} 
en los sistemas antiguos y en los sistemas de las Fuerzas Armadas y de Orden. ${ }^{15}$ Dado que los trabajadores dependientes (que asignaremos al grupo 2) deben pagar ISC si corresponde, su ingreso líquido es:

$$
\text { Monto líquido }_{2}=\text { Base tributable }_{2}(1-\text { tas } a)+\text { Descuento tramo }
$$

Donde tasa corresponde a la tasa de ISC que deben pagar por sus ingresos y Descuento tramo corresponde al descuento asociado a dicho tramo por la consideración del tramo exento y las menores tasas pagadas en intervalos inferiores.

Con esto, la base tributable del trabajador del grupo 2 corresponde a:

$$
\text { Base tributable }_{2}=\frac{\text { Monto líquido }_{2}-\text { Descuento tramo }}{(1-\text { tas } a)}
$$

Por último, a aquellos trabajadores que no cotizan como dependientes pero que sí están contratados (grupo 3), les asignaremos su base tributable siguiendo la misma estructura anterior, es decir:

$$
\text { Base tributable }_{3}=\frac{\text { Monto líquido }_{3}-\text { Descuento tramo }}{(1-\text { tas } a)}
$$

A aquellos trabajadores que no entregan boleta, no cotizan como dependientes ni tienen contrato de trabajo los clasificaremos en el grupo 4 y su base tributable corresponderá a 0 .

El Gráfico 6 muestra en resumen la división que generamos y la Tabla 2 muestra la cantidad de trabajadores asociados a dichas clasificaciones.

De la Tabla 1 se puede observar que la cantidad de personas que recibieron ingresos del trabajo en el período corresponde a 8.192.714, lo que está razonablemente cercano a las 8.386 .827 personas que recibieron ingresos del trabajo de acuerdo con la Encuesta Suplementaria de Ingresos (ESI) del Instituto Nacional de Estadísticas en el trimestre octubre-

\footnotetext{
${ }^{15}$ Esto es posible ya que las preguntas sobre cotizaciones previsionales de la encuesta Casen incluyen un ítem en el cual se pregunta si se cotiza en el sistema de pensiones de manera dependiente o independiente. Concretamente nos referimos a la pregunta o29: "¿Cotizó durante el mes pasado en algún sistema previsional (sistema de pensiones)?".
} 
Gráfico 6. CLASIFICACIONES DE LOS TRABAJADORES

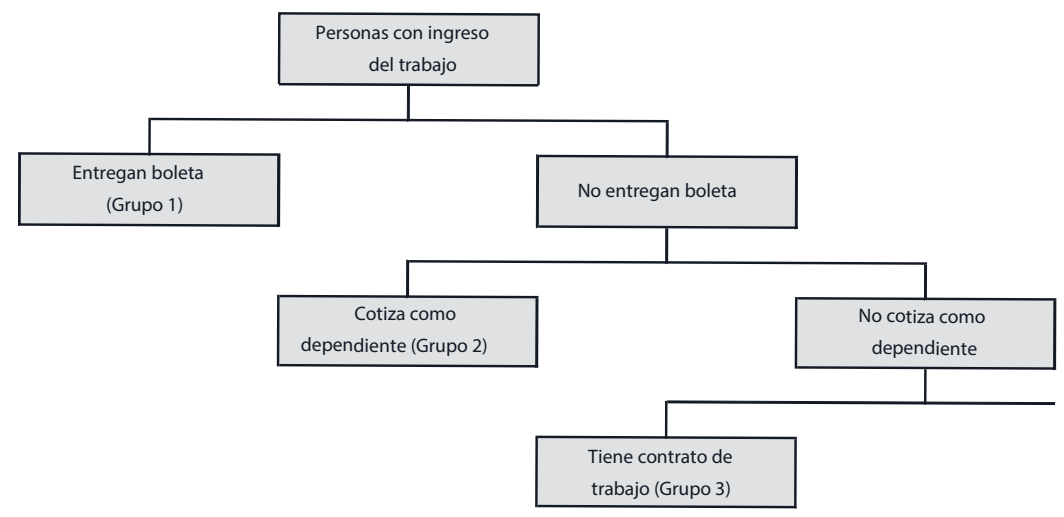

Fuente: Elaboración propia.

Tabla 2. CANTIDAD DE PERSONAS CON INGRESOS DEL TRABAJO POR GRUPO

\begin{tabular}{lcc}
\hline Grupo & Cantidad de trabajadores & Porcentaje del total (\%) \\
\hline 1 & 878.430 & 10,7 \\
2 & 4.785 .055 & 58,4 \\
3 & 366.470 & 4,5 \\
4 & 2.162 .759 & 26,4 \\
Total & 8.192 .714 & 100,0 \\
\hline
\end{tabular}

Nota: La variable utilizada corresponde a ytrabajocor.

Fuente: Elaboración propia sobre la base de datos de la encuesta Casen (2017).

diciembre de $2017 .{ }^{16}$ Es muy importante mencionar que recibir ingresos del trabajo no es necesariamente equivalente a estar ocupado, ya que mientras lo primero requiere simplemente que las personas reporten ingresos por esta vía, lo segundo requiere que las personas hayan trabajado al menos una hora en la semana de referencia $y$, en caso contrario, que tengan un empleo del que se encuentran ausentes por un motivo tempo-

\footnotetext{
${ }^{16}$ La ESI es la principal encuesta para medir ingresos del trabajo utilizada en Chile, ya que corresponde a un módulo de la Encuesta Nacional de Empleo (ENE) que se aplica en el trimestre de octubre-diciembre de cada año.
} 
ral. ${ }^{17} \mathrm{~A}$ modo de ejemplo, alguien que haya recibido un pago por un trabajo fuera de la semana de referencia y que no tenga un empleo, no será clasificado como ocupado, pero sí reportará ingresos del trabajo, de forma que no todas las personas que declaran ingresos del trabajo estarán ocupadas. Sin embargo, como el aspecto que nos interesa es la tributación de los ingresos, y no que se cumplan los requisitos para ser ocupados, trabajaremos sobre la base de quienes reportan ingresos del trabajo.

Adicionalmente, las personas que reportan ingresos del trabajo de manera informal de acuerdo con nuestra clasificación corresponden a 2.162.759 personas, mientras que la ESI indica que 2.611.794 personas declaran ingresos del trabajo y no cumplen con las condiciones de formalidad laboral del INE. ${ }^{18} 19$ De esta forma, nuestra estimación logra capturar cerca del $83 \%$ de esta magnitud. La Tabla 3 presenta las bases tributables promedio de los distintos grupos de trabajadores, si es que aplicamos las fórmulas expresadas anteriormente.

Tabla 3. BASES TRIBUTABLES PROMEDIO POR GRUPO DE TRABAJADORES

\begin{tabular}{|c|c|c|c|}
\hline Grupo & Cantidad de trabajadores & Base tribut & able promedio \\
\hline 1 & 878.430 & $\$$ & 904.383 \\
\hline 2 & 4.785 .055 & $\$$ & 660.323 \\
\hline 3 & 366.470 & $\$$ & 526.481 \\
\hline 4 & 2.162 .759 & $\$$ & - \\
\hline
\end{tabular}

Fuente: Elaboración propia sobre la base de datos de la encuesta Casen (2017).

De esta forma, las personas que pertenecen a los grupos 1, 2 y 3 deberían ser consideradas para pago de ISC, lo que corresponde a un total de 6.029.955. ${ }^{20}$ Esta cifra puede compararse con los informes del SII, en

\footnotetext{
${ }^{17}$ En el cuestionario Casen, esto corresponde a las preguntas o1, 02 y o3.

${ }^{18}$ Parte de esta diferencia se explica porque nuestra clasificación solo estudia la formalidad del trabajador, mientras que la clasificación del INE también considera la formalidad del empleador.

${ }^{19}$ La cifra se obtiene de restar al total de 8.386 .827 personas que declaran haber recibido ingresos del trabajo, aquellos 5.775 .033 que cumplen las condiciones para ser ocupados formales.

${ }^{20}$ Para efectos de este trabajo, los pensionados no serán considerados para efectos del ISC, ya que no son clasificables en los grupos mencionados. De esta forma, sus ingresos serán considerados como 'otros ingresos' y solo se les aplicará IGC. Esto se debe principalmente a la dificultad para determinar el monto de la pensión en la encuesta Casen, debido a los diversos componentes que la integran.
} 
donde se menciona la cantidad de personas que quedan afectas a cada impuesto. En este caso, el SIl informa que en 2017 la cantidad de trabajadores que declararon rentas del trabajo se ubicó en 6.619.603, vale decir, nuestra aproximación permite identificar a un $91 \%$ de los trabajadores que reportan rentas del trabajo. ${ }^{21}$

Otra manera de comprobar qué tan cercanas están nuestras estimaciones de la realidad, es comparando los ingresos imponibles promedio de aquellos cotizantes dependientes, con los ingresos imponibles promedio para dichos grupos reportados por la Superintendencia de Pensiones (SP). Para ello se toman las bases tributables de aquellos trabajadores que pertenecen al grupo 2 , y agregamos a dicha base un monto correspondiente por seguro de salud y por pago de cotizaciones previsionales. Si asumimos que el pago por concepto de seguro de salud y de cotización previsional corresponde al $7 \%$ y $11,14 \%$ de la base imponible, respectivamente, ${ }^{22}$ entonces la base imponible de los trabajadores puede calcularse como:

$$
\text { Base imponible }=\frac{\text { Base tributable }}{1-0,0700-0,1114}=\frac{\text { Base tributable }}{0,8186}
$$

Así, la encuesta Casen nos entrega que hay 5.134.445 trabajadores que cotizaron de forma dependiente ${ }^{23}$ sin embargo, solo podemos estimar los ingresos imponibles de 4.952.734, de los cuales obtenemos un ingreso imponible promedio de $\$ 821.591 .{ }^{24}$ Por su parte, la Superintendencia de Pensiones reporta que entre noviembre de 2017 y enero de 2018 hubo en promedio 5.224.923 trabajadores que cotizaron de forma dependiente y que tuvieron ingresos imponibles promedio por $\$ 774.554$.

Lo anterior implica que nuestras estimaciones a partir de la encuesta Casen tienen errores cercanos al $5 \%$ y al $6 \%$, tanto para la cantidad de

${ }^{21}$ Esta información se encuentra disponible en http://www.sii.cl/sobre_el_sii/estadisticas_ de_personas_naturales.html [20 de julio 2020].

${ }^{22}$ Esto se calculó a través del 10\% que se aporta a las cuentas individuales más el promedio simple de las comisiones de las seis AFP abiertas existentes en diciembre de 2017: Capital, Cuprum, Habitat, Modelo, Planvital y Provida.

${ }^{23}$ Notar que esto es mayor a los 4.785 .055 trabajadores del grupo 2, ya que también hay personas que entregan boletas (grupo 1) y que declaran cotizar como dependientes.

${ }^{24}$ Esto ocurre porque no todos los trabajadores que declaran sus condiciones laborales, como tenencia de contrato, contestan la pregunta de ingresos. 
cotizantes dependientes como para sus ingresos imponibles, respectivamente. Lo anterior es también una muestra de que nuestra aproximación captura de buena forma la gran mayoría de los ingresos del trabajo de las personas.

Posteriormente, procedemos a calcular el ISC. Esto se hace siguiendo la estructura del impuesto de segunda categoría del mes de diciembre de 2017 que está presentado en la Tabla 4. La Tabla 5 muestra el pago promedio mensual y anual por concepto de ISC. Es relevante recordar que la retención del $10 \%$ de los pagos a honorarios es considerada recaudación de ISC por parte del SII.

Tabla 4. IMPUESTO DE SEGUNDA CATEGORÍA (DICIEMBRE 2017)

\begin{tabular}{|c|c|c|c|c|c|}
\hline \multicolumn{6}{|c|}{ Impuesto de Segunda Categoría (diciembre 2017) } \\
\hline \multirow{2}{*}{$\begin{array}{l}\text { Tramo } \\
1\end{array}$} & \multicolumn{2}{|c|}{ Montos de renta imponible } & \multirow{2}{*}{$\begin{array}{c}\text { Tasa (\%) } \\
0,0\end{array}$} & \multicolumn{2}{|c|}{ Cantidad a rebajar } \\
\hline & $\$$ & $\$ 634.122,00$ & & $\$$ & - \\
\hline 2 & $\$ 634.122,01$ & $\$ 1.409 .160,00$ & 4,0 & $\$$ & $25.364,88$ \\
\hline 3 & $\$ 1.409 .160,01$ & $\$ 2.348 .600,00$ & 8,0 & $\$$ & $81.731,28$ \\
\hline 4 & $\$ 2.348 .600,01$ & $\$ 3.288 .040,00$ & 13,5 & $\$$ & $210.904,28$ \\
\hline 5 & $\$ 3.288 .040,01$ & $\$ 4.227 .480,00$ & 23,0 & $\$$ & $523.268,08$ \\
\hline 6 & $\$ 4.227 .480,01$ & $\$ 5.636 .640,00$ & 30,4 & $\$$ & $836.101,60$ \\
\hline 7 & $\$ 5.636 .640,01$ & y más & 35,0 & & $1.095 .387,04$ \\
\hline
\end{tabular}

Fuente: Elaboración propia sobre la base de datos de la encuesta Casen (2017).

Un primer resultado de este ejercicio es notar que la recaudación por concepto de impuesto de segunda categoría rondaría los US\$3.752 millones. ${ }^{25}$ Esta cifra corresponde al $96 \%$ de los US\$3.920 millones declarados por el SII en el año $2017 .^{26}$

Un segundo resultado de este ejercicio es notar que cerca de un $26,4 \%$ de las personas que reportan ingresos del trabajo no tributarían (grupo 4). Este grupo, conformado por quienes no tienen contrato, no dan boleta y tampoco cotizan. El grupo corresponde a 2,16 millones de

\footnotetext{
${ }^{25}$ Desde aquí en adelante, todas las medidas en dólares serán en base al dólar del año 2017. El tipo de cambio considerado es el valor anual reportado por el Banco Central de Chile, que corresponde a $\$ 649,33$ por US\$1.

${ }^{26}$ El SIl declara que por retenciones de impuestos de segunda categoría se recaudaron $\$ 2.545 .767$ millones, lo que a un tipo de cambio de $\$ 649,33$ por US\$1, entrega la cantidad mencionada.
} 
trabajadores, quienes aproximadamente tienen un ingreso imponible promedio de $\$ 262$ mil, lo que los identifica como un grupo vulnerable. Si este grupo tributara pagaría un promedio de $\$ 3.924$ mensuales, lo que significaría una recaudación anual adicional de US\$157 millones.

Tabla 5. PAGOS PROMEDIO DE ISC MENSUAL Y ANUAL POR CLASIFICACIÓN DE TRABAJADORES

\begin{tabular}{lccc}
\hline Grupo & Cantidad de trabajadores & Pago promedio mensual & Pago promedio anual \\
\hline 1 & 878.430 & $\$ 90.438$ & $\$ 1.085 .259$ \\
2 & 4.785 .055 & $\$ 24.626$ & $\$ 295.514$ \\
3 & 366.470 & $\$ 15.632$ & $\$ 187.581$ \\
4 & 2.162 .759 & $\$-$ & $\$$ \\
\hline
\end{tabular}

Fuente: Elaboración propia sobre la base de datos de la encuesta Casen (2017).

Con el ejercicio anterior, hemos determinado en base a la Casen 2017 los ingresos imponibles del trabajo. Ahora procederemos a determinar los ingresos imponibles anuales totales. Para ello, en primer lugar se calculan los ingresos que no provienen del trabajo ni de subsidios y se anualizan. ${ }^{27}$ Posteriormente, sumaremos a este monto los ingresos imponibles anuales de los trabajadores. ${ }^{28}$ En caso de que los trabajadores pertenezcan al grupo 4, sus ingresos imponibles del trabajo serán cero. En este ejercicio estamos haciendo el supuesto de que todos los ingresos adicionales al trabajo son reportados al SII. Si bien este supuesto parece poco realista, no hay manera de determinar qué ingresos son reportados y cuáles no. Adicionalmente, pensamos que mientras mayor sea el monto de los otros ingresos será más difícil que el SIl no tenga conocimiento de ellos, de forma que esto no debería afectar en forma significativa nuestra recaudación estimada.

Es importante tener en cuenta que hay diversos créditos al IGC. En primer lugar está el pago del ISC para quienes corresponde pagarlo. Adicional a este, se encuentra el crédito por los dividendos y utilidades retiradas de empresas, el cual corresponde al impuesto de primera categoría pagado por dichos ingresos. Dado que en 2017 el impuesto de

\footnotetext{
${ }^{27}$ Estos ingresos se pueden calcular de dos formas que son equivalentes. Una forma corresponde a la diferencia entre los ingresos autónomos y los ingresos del trabajo. La otra forma corresponde a la diferencia entre los ingresos totales y la suma de los ingresos del trabajo y las transferencias monetarias.

${ }^{28}$ Este es el monto imponible mensual por 12.
} 
primera categoría era de 25,5\%, para obtener el crédito correspondiente, tomaremos la suma de los ingresos declarados por utilidades retiradas y dividendos de acciones y aplicaremos la siguiente fórmula:

$$
\text { Crédito }=\left(\frac{(\text { Retiro de utilidades }+ \text { Dividendos de acciones })}{0,745} \times 0,255\right) \times 0,65
$$

Dividimos por 0,745 para obtener la totalidad de las utilidades generadas y luego multiplicamos por 0,255 para obtener el pago del impuesto de primera categoría. Finalmente, multiplicamos todo por 0,65, ya que representa la fracción del pago de impuestos que es utilizable como crédito en el sistema parcialmente integrado. ${ }^{29}$ Para efectos de este trabajo asumiremos que todas las firmas son semiintegradas, ya que por su naturaleza son las firmas de mayores ingresos y, por ende, las más relevantes en términos de recaudación.

Adicionalmente, hay otros créditos al IGC como los intereses pagados por concepto de créditos hipotecarios y una proporción del gasto en educación preescolar, básica, diferencial y media. Sin embargo, no tenemos forma de saber estos montos, por lo que no se incluyen en nuestras simulaciones. Por otro lado, hay ingresos del capital como los intereses ganados por depósitos a plazo que no se consideran renta si es que el contribuyente tiene solo rentas gravadas en ciertas condiciones ${ }^{30}$ y los intereses no exceden las 20 UTM. ${ }^{31}$ Dado que la encuesta Casen no permite identificar con certeza la procedencia de la renta, nuestra simulación gravará todo este tipo de ingresos, lo cual disminuirá el monto a devolver por concepto de IGC.

Por otro lado, también hay consideraciones que disminuyen el ingreso imponible a considerar para efectos de calcular el IGC. La principal de estas especifica que los trabajadores que entregan boleta y que tra-

\footnotetext{
${ }^{29}$ El sistema semiintegrado comenzó a regir el 1 de enero de 2017. Este obliga a tributar bajo este esquema a las sociedades anónimas cerradas y abiertas, a las sociedades en comanditas por acción y a las sociedades de personas o por acciones que cuenten al menos con un socio o accionista persona jurídica. La tasa del $25,5 \%$ solo aplicó a las empresas que estuviesen en el sistema semiintegrado, mientras que las que se acogieron al sistema de renta atribuida tenían una tasa de $25 \%$. Disponible en: http s://www.bcn.cl/leyfacil/recurso/ reforma-tributaria-2014 [23 de junio 2020].

${ }^{30}$ En particular, que sean gravadas por el artículo $42, N^{\circ} 1$, correspondiente a los sueldos, 0 22 , correspondiente a los contribuyentes pequeños.

${ }^{31}$ Límite que además considera los dividendos.
} 
bajan de forma independiente (una parte de nuestro grupo 1) pueden rebajar un 30\% de los ingresos imponibles como presunción de gasto para generar la renta restante. Esta rebaja es considerada en nuestras estimaciones, considerando solo el $70 \%$ de los ingresos del trabajo como base imponible en el caso de que el trabajador pertenezca al grupo 1 y además sea independiente. ${ }^{32}$ Es importante notar que esta rebaja tiene un máximo de 15 UTA, que en diciembre de 2017 correspondía a $\$ 8.454 .960$. La Tabla 6 muestra el impuesto global complementario para el año 2018 (ingresos anuales de 2017).

Tabla 6. DISTRIBUCIÓN DE TRAMOS AL IMPUESTO GLOBAL COMPLEMENTARIO AÑO 2018 (INGRESOS DE 2017)

\begin{tabular}{|c|c|c|c|c|c|}
\hline \multicolumn{6}{|c|}{ Impuesto Global Complementario 2018 (Rentas de 2017) } \\
\hline \multirow{2}{*}{$\frac{\text { Tramo }}{1}$} & \multicolumn{2}{|c|}{ Montos de renta imponible } & \multirow{2}{*}{$\begin{array}{c}\text { Tasa (\%) } \\
0,0\end{array}$} & \multicolumn{2}{|c|}{ Cantidad a rebajar } \\
\hline & $\$$ & $\$ 7.609 .464,00$ & & $\$$ & - \\
\hline 2 & $\$ 7.609 .464,01$ & $\$ 16.909 .920,00$ & 4,0 & $\$$ & $304.378,56$ \\
\hline 3 & $\$ 16.909 .920,01$ & $\$ 28.183 .200,00$ & 8,0 & $\$$ & $980.775,36$ \\
\hline 4 & $\$ 28.183 .200,01$ & $\$ 39.456 .480,00$ & 13,5 & $\$$ & $2.530 .851,36$ \\
\hline 5 & $\$ 39.456 .480,01$ & $\$ 50.729 .760,00$ & 23,0 & & $6.279 .216,96$ \\
\hline 6 & $\$ 50.729 .760,01$ & $\$ 67.639 .680,00$ & 30,4 & & $10.033 .219,20$ \\
\hline 7 & $\$ 67.639 .680,01$ & y más & 35,0 & $\$$ & $13.144 .644,48$ \\
\hline
\end{tabular}

Fuente: Servicio de Impuestos Internos (SII).

La Tabla 7 muestra cómo, en base a la Casen 2017, se distribuyen las personas con ingresos imponibles entre los distintos tramos y los pagos promedio que realizan (si el valor es positivo) o que les es devuelto (si es negativo).

\footnotetext{
32 Esta condición de independencia se verifica en la pregunta o15 de la encuesta Casen, que pregunta "En su empleo o trabajo principal, ¿usted trabaja cómo?" y las alternativas son:

1. Patrón o empleador

2. Trabajador por cuenta propia

3. Empleado u obrero del sector público

4. Empleado u obrero de empresas públicas

5. Empleado u obrero del sector privado

6. Servicio doméstico puertas adentro

7. Servicio doméstico puertas afuera

8. FF.AA. y de Orden

9. Familiar no remunerado
} 
Tabla 7. DISTRIBUCIÓN DE PERSONAS EN DISTINTOS TRAMOS DEL IGC

\begin{tabular}{lrcrr}
\hline Tramo & Personas & Porcentaje (\%) & \multicolumn{2}{c}{ Paga en promedio } \\
\hline 1 & 3.095 .892 & 77,8 & $\$$ & -80.162 \\
2 & 541.701 & 13,6 & $\$$ & -335.457 \\
3 & 184.815 & 4,6 & $\$$ & -361.705 \\
4 & 75.866 & 1,9 & $\$$ & -90.673 \\
5 & 28.625 & 0,7 & $\$$ & 642.695 \\
6 & 26.130 & 0,7 & $\$$ & 2.005 .845 \\
7 & 28.706 & 0,7 & $\$ 11.338 .159$ \\
\hline Total & 3.981 .735 & 100,0 & $\$$ & -26.956 \\
\hline
\end{tabular}

Fuente: Elaboración propia sobre la base de datos de la encuesta Casen (2017).

Como se puede observar, se obtiene que 3.981.735 personas tendrían rentas afectas a IGC. En este caso, el SII reporta que en 2017 las personas con rentas afectas a este fueron 3.321.462, de forma que nuestra aproximación sobreestima en un $20 \%$ la cantidad real. Atribuimos este factor, como mencionamos anteriormente, al supuesto de asumir que todas las rentas no relacionadas al trabajo son declaradas al SII.

Estas estimaciones generan que un $77,8 \%$ de las personas que declara ingresos está exenta de pagar el IGC. Sin embargo, hasta el tramo 4, los contribuyentes presentan más créditos que impuestos por pagar $y$, por lo tanto, reciben devoluciones. Este hecho genera que el IGC en términos agregados devuelva más dinero del que recauda. Así, nuestras estimaciones indican que el IGC devolvió en términos netos en 2017 del orden de US\$165 millones, mientras que los datos oficiales del SII indican que se devolvieron en términos netos US\$295 millones, lo que implica que podemos explicar un $56 \%$ de las devoluciones con nuestra simulación. Atribuimos esta cantidad a la incapacidad de la encuesta Casen para permitir identificar más créditos a este impuesto.

Sin embargo, a pesar de los desajustes en el IGC, de acuerdo a nuestras estimaciones los impuestos a las personas en Chile sumaban una recaudación de US\$3.586 millones en 2017, lo que representa un 1,29 puntos del PIB de ese año. ${ }^{33}$ Por su parte, el SII informa que la recaudación neta entre el ISC y el IGC corresponde a US\$3.625 millones, lo que

\footnotetext{
${ }^{33}$ Considerando el PIB de Chile del año 2017 a precios corrientes de US\$277.746 millones.
} 
corresponde a 1,31 puntos del PIB. ${ }^{34}$ Así, en el agregado logramos, con nuestra metodología, capturar el 99\% de la recaudación del impuesto a la renta personal informado por el SII. Nos parece que capturar este porcentaje de la recaudación total a partir de los datos de la Casen es suficientemente cercano a la realidad para que los ejercicios en la sección que sigue sean también buenas aproximaciones de esta.

Sin embargo, es importante aclarar que al momento de aplicar los esquemas de los distintos países no vamos a hacer ajustes por cambios en la elasticidad de recaudación. ${ }^{35}$ De esta manera, supondremos que la forma en que los contribuyentes se comportan no cambiará junto con los cambios de tasas. Si bien la literatura al respecto muestra que los contribuyentes son sensibles a cambios en las tasas (e.g. Hutton y Lambert 1980; Breeden y Hunter 1985; Creedy y Gemmell 2004; Kopczuk 2005), modelar la forma en que los contribuyentes chilenos podrían responder frente a cada uno de los cambios tributarios, excede el alcance de este artículo.

Por último, tenemos que ver la distribución de ingreso que generan los ingresos autónomos y los ingresos monetarios de los hogares. Siguiendo la metodología de Jolliffe y Krushelnytskyy (2000), ${ }^{36}$ tenemos inicialmente que el coeficiente de Gini de los ingresos autónomos tiene una media de 0.502 y el de los ingresos monetarios tiene una media de 0.489. Vale destacar que la diferencia entre ambos coeficientes de Gini es estadísticamente significativa, por lo que las transferencias monetarias — que suman US\$3.333 millones— ${ }^{37}$ sí logran alterar la distribución del ingreso en Chile, aunque en forma muy menor. Sin embargo, estos coeficientes no consideran el pago del IGC que acabamos de incluir. Para recalcular los nuevos coeficientes de Gini, calcularemos los nuevos ingresos autónomos y monetarios de la siguiente forma:

Nuevo ingreso autónomo = Base tributable + Otros ingresos - Pago ISC $-\left(\frac{\text { Pago IGC }}{12}\right)$

Nuevo ingreso monetario

$$
\begin{aligned}
& =\text { Base tributable + Otros ingresos - Pago ISC }-\left(\frac{\text { Pago IGC }}{12}\right) \\
& + \text { Transferencias monetarias }
\end{aligned}
$$

\footnotetext{
${ }^{34}$ El SIl informa que el neto de ambos impuestos corresponde a 1,33 puntos del PIB, pero eso se debe a que consideran un PIB en 2017 de US\$272.572 millones.

${ }^{35}$ La elasticidad de recaudación se refiere a la medida en que cambian los ingresos tributarios al cambiar las tasas de los impuestos.

${ }^{36}$ En Stata, esta metodología se puede replicar a través del comando inequerr.

${ }^{37}$ Esta es la suma de las transferencias reportadas por la encuesta Casen (2017).
} 
La base tributable considera los ingresos del trabajo ajustados según las distintas formas que expusimos anteriormente. En el caso de los trabajadores del grupo 4, su base imponible es cero, pero se les considera el ingreso que obtienen del trabajo, ya que pretendemos saber los ingresos con los que cuentan, más allá de que estos tributen o no. El pago del IGC se divide por 12 para obtener un pago mensualizado. Con esto, recalculamos los coeficientes de Gini y obtenemos medias de 0,499 y 0,486 para los ingresos autónomos y monetarios, respectivamente, siendo ninguno de estos estadísticamente diferente de sus pares que no incluyen el IGC.

\section{Impuesto a la renta en Chile bajo la estructura de tramos de otros países}

En esta sección calculamos, en base a la distribución de ingresos de las personas que se determinó en la sección anterior, cuánto se recaudaría por este impuesto si Chile tuviera una estructura del impuesto a la renta personal de Australia, España, Noruega y Nueva Zelanda. Se hace para los tramos, ajustando y no ajustando por PPC. Además se obtiene cómo cambiarían los coeficientes Gini en cada caso.

\section{Australia}

Tal como se mencionó previamente, la conversión de la estructura de impuesto a la renta de las personas de otro país a Chile se puede hacer de dos formas. La primera es simplemente usar el tipo de cambio nominal, que en el caso de Australia correspondía en 2017 a $\$ 497,2$ por AUD\$1. ${ }^{38}$ La segunda es usar el tipo de cambio corregido por paridad de poder de compra (PPC), que para Australia correspondía en 2017 a \$280,5 por AUD\$1. ${ }^{39}$ Esto significa que un dólar australiano compraba en 2017 en Chile casi dos veces más bienes que los que compraba en Australia. La

\footnotetext{
$\overline{38}$ Esto se obtiene a partir de datos del Banco Mundial a través del tipo de cambio nominal del dólar norteamericano para el dólar australiano y el peso chileno. Para el resto de los países se realizará el equivalente.

${ }^{39}$ Esto se obtiene a partir de datos del Banco Mundial a través del tipo de cambio ajustado por PPC del dólar internacional para el dólar australiano y el peso chileno. Para el resto de los países se realizará el equivalente.
} 
primera medición, entonces, está referida al tipo de cambio nominal, y la segunda, a la capacidad de compra. Realizaremos las simulaciones con ambos tipos de cambio. La Tabla 8 muestra la estructura impositiva para los ingresos anuales de las personas en Australia.

Tabla 8. ESTRUCTURA IMPOSITIVA PARA LOS INGRESOS DE LAS PERSONAS EN AUSTRALIA

\begin{tabular}{lrccc}
\hline \multicolumn{5}{c}{ Australia - Gobierno central ${ }^{*}$} \\
\hline Nivel de ingreso & Tasa $^{* *}$ \\
\hline AUD & - & AUD & 18.200 & $0,0 \%$ \\
AUD & 18.200 & AUD & 37.000 & $19,0 \%$ \\
AUD & 37.000 & AUD & 87.000 & $32,5 \%$ \\
AUD & 87.000 & AUD & 180.000 & $37,0 \%$ \\
AUD & 180.000 & o más & $45,0 \%$ \\
\hline
\end{tabular}

Nota*: Además, existe un crédito no reembolsable denominado LITO, ${ }^{40}$ que corresponde a AUD 445 para ingresos menores a AUD 37.000 y cae 1,5 centavos para cada AUD 1 por ingresos mayores a AUD 37.000 .

Nota**: Adicionalmente a la tasa de ingresos totales, existe una tasa de $2 \%$ para todos los ingresos que se destina a pagar el sistema de salud. Esta tasa puede variar según composiciones del hogar y características de las personas. Dado que este es un impuesto de seguridad social, no será considerado en nuestra simulación.

Fuente: Elaboración propia en base a datos de la OCDE.

La Tabla 9 muestra el ajuste de la estructura tributaria siguiendo el tipo de cambio nominal (es decir, se multiplican los tramos de la Tabla 8 por 497,2).

Adicionalmente, el LITO máximo corresponde a \$221.250 para ingresos menores a $\$ 18.396 .060$, cayendo posteriormente $\$ 1,5$ por cada $\$ 100$ adicionales, por lo que se agota en ingresos de $\$ 33.146 .050$. Este descuento se aplicará a quienes corresponda pagar IGC, considerando que no es reembolsable.

Por su parte, para el tipo de cambio ajustado por PPC, la Tabla 10 refleja la estructura impositiva con la que se gravarán las rentas imponibles anuales totales.

\footnotetext{
${ }^{40}$ Low Income Tax Offset en inglés. Notar que también existe el LMITO, que incluye ingresos medios, pero este empieza a ser considerado para los ingresos a partir de 2018-2019, por lo que no es considerado en este ejercicio.
} 
Tabla 9. ESTRUCTURA IMPOSITIVA PARA LOS INGRESOS IMPONIBLES ANUALES DE LAS PERSONAS, SIGUIENDO A AUSTRALIA POR TIPO DE CAMBIO NOMINAL

\begin{tabular}{ccccc}
\hline \multicolumn{5}{c}{ Chile - Australia Gobierno central (Nominal) } \\
\hline \multicolumn{5}{c}{ Nivel ingreso } \\
\hline$\$$ & - & $\$ 9.048 .873$ & Tasa (\%) \\
$\$$ & 9.048 .873 & $\$ 18.396 .060$ & 0,0 \\
$\$$ & 18.396 .060 & $\$ 43.255 .600$ & 19,0 \\
$\$$ & 43.255 .600 & $\$ 89.494 .345$ & 32,5 \\
$\$$ & 89.494 .345 & & o más & 37,0 \\
\hline
\end{tabular}

Fuente: Elaboración propia.

Tabla 10. ESTRUCTURA IMPOSITIVA PARA LOS INGRESOS IMPONIBLES ANUALES DE LAS PERSONAS, SIGUIENDO A AUSTRALIA POR TIPO DE CAMBIO AJUSTADO POR PPC

\begin{tabular}{lrrrr}
\hline \multicolumn{4}{c}{ Chile - Australia Gobierno central (PPC) } \\
\hline \multicolumn{5}{c}{ Nivel ingreso } \\
\hline$\$$ & - & $\$$ & 5.105 .733 & Tasa (\%) \\
$\$$ & 5.105 .733 & $\$$ & 10.379 .787 & 0,0 \\
$\$$ & 10.379 .787 & $\$$ & 24.406 .527 & 19,0 \\
$\$$ & 24.406 .527 & $\$$ & 50.496 .262 & 32,5 \\
$\$$ & 50.496 .262 & & o más & 37,0 \\
\hline
\end{tabular}

Fuente: Elaboración propia.

Adicionalmente, el LITO máximo corresponde a \$124.838 para ingresos menores a $\$ 10.379 .787$, cayendo posteriormente $\$ 1,5$ por cada $\$ 100$ adicionales, por lo que se agota en ingresos de $\$ 18.702 .317$.

Cabe destacar que una diferencia relevante entre Chile y Australia es la cantidad de tramos y la pendiente entre tramos. Mientras Chile tiene 7 tramos con tasas de $0 \%, 4 \%, 8 \%, 13,5 \%, 23 \%, 30,4 \%$ y $35 \%$, respectivamente, el sistema australiano tiene solo 5 tramos con tasas de $0 \%, 19 \%$, $32,5 \%, 37 \%$ y $45 \%$.

Si usamos el tipo de cambio nominal para hacer la equivalencia, se recaudarían US\$6.470 millones por concepto de ISC y US\$1.144 millones por concepto de IGC. La recaudación positiva del IGC se debe a que la retención de $10 \%$ no es un monto significativo para aquellos que pagan 
impuestos, pues la tasa sube rápidamente y supera la retención en niveles bajos de ingreso, a diferencia del caso chileno.

Esto dejaría a la recaudación por concepto de impuestos a las personas en un total de US\$7.615 millones (ascendiendo a un 2,7\% del PIB), lo que implica una recaudación adicional a lo que se recaudó en 2017 en Chile de US\$4.029 millones anuales, o un 1,5\% del PIB. Además, esto se traduce en un coeficiente Gini del ingreso monetario de 0,468 , siendo 0,018 puntos menor que en el escenario base.

Por su parte, si usamos el tipo de cambio ajustado por PPC se recaudarían US\$9.520 millones por concepto de ISC, y US\$2.405 millones por concepto de IGC. Al igual que en el caso anterior, la recaudación positiva del IGC se debe a que la retención del $10 \%$ no es un monto significativo para aquellos que pagan impuestos.

Esto dejaría a la recaudación por concepto de impuestos a las personas en un total de US\$11.925 millones (ascendiendo a un 4,3\% del PIB), lo que implica una recaudación adicional de US\$8.339 millones anuales o $3 \%$ del PIB. Además, esto se traduce en un coeficiente Gini del ingreso monetario de 0,455 , siendo 0,031 puntos menor que en el escenario base.

La pregunta que sigue es la siguiente: ¿qué se hace con la mayor recaudación? Evidentemente, ello podría afectar la distribución del ingreso. Se supone que la forma en que estas mayores transferencias llegan a las personas es igual a la forma como lo hacen hoy, por no tener una mejor manera de hacerlo. Pero, evidentemente, si ellas fueran mejor focalizadas, el coeficiente de Gini obtenido podría ser más bajo. En ese sentido, se puede interpretar el resultado como el límite inferior en cuanto a posible disminución en la desigualdad. Así, entonces, se presentan cuatro escenarios posibles de utilización de la recaudación adicional:

1) No se aumentan las transferencias.

2) Se aumentan las transferencias usando un $33 \%$ de la recaudación adicional.

3) Se aumentan las transferencias usando un $67 \%$ de la recaudación adicional.

4) Se aumentan las transferencias usando la totalidad de la recaudación adicional. 
Los resultados se muestran en la Tabla 11. Se observa que en el caso extremo (todo se usa en más transferencias) el Gini podría bajar a 0,45 (en el ejercicio con tipo de cambio nominal) o a 0,42 (tipo de cambio a PPC). Para ilustrar qué sucede con la distribución de los ingresos por decil, en el Gráfico 7 se muestra qué sucede con los ingresos promedio de cada decil cuando el ejercicio se hace con ambos tipos de cambio y se distribuye como transferencia un tercio de la mayor recaudación. Queda en evidencia que la diferencia proviene del mayor ingreso monetario de los deciles más bajos y la disminución en los deciles más altos. Pero, sin lugar a dudas, el mayor efecto proviene de la importante disminución en el ingreso monetario del decil de mayores ingresos. Adicionalmente, vale insistir en que este ejercicio consiste en simular las distribuciones de ingresos si es que se copiaran los tramos y tasas impositivas de distintos países. Lo anterior no implica que el camino de política sugerido sea copiarlos sin hacer ajustes. Por ejemplo, puede no ser socialmente deseable que los deciles VII y VIII disminuyan sus ingresos; por lo tanto, al momento de planificar una reforma tributaria a los ingresos de las personas, es importante generar escenarios en que ello no ocurra. También hay que tener en consideración que este resultado en particular es dependiente de la forma en que aumentamos las transferencias.

Tabla 11. RESUMEN DE COEFICIENTES DE GINI Y RECAUDACIÓN PARA EL ESTADO DE ACUERDO CON EL AUMENTO DE TRANSFERENCIAS

\begin{tabular}{|c|c|c|c|c|}
\hline \multirow{2}{*}{$\begin{array}{l}\text { País } \\
\text { Australia }\end{array}$} & \multicolumn{2}{|c|}{ Nominal } & \multicolumn{2}{|c|}{ PPC } \\
\hline & $\begin{array}{c}\text { Gini } \\
\text { monetario }\end{array}$ & $\begin{array}{l}\text { Recaudación } \\
\text { adicional }\end{array}$ & $\begin{array}{c}\text { Gini } \\
\text { monetario }\end{array}$ & $\begin{array}{c}\text { Recaudación } \\
\text { adicional }\end{array}$ \\
\hline Transferencias base de Chile & 0,468 & \multirow{3}{*}{$\begin{array}{l}\text { USD } 4.029 \\
\text { millones }\end{array}$} & 0,455 & \multirow{4}{*}{$\begin{array}{l}\text { USD } 8.339 \\
\text { millones }\end{array}$} \\
\hline $\begin{array}{l}\text { Aumentadas en } 33 \% \text { recaudación de la } \\
\text { adicional }\end{array}$ & 0,460 & & 0,439 & \\
\hline $\begin{array}{l}\text { Aumentadas en } 67 \% \text { recaudación de la } \\
\text { adicional }\end{array}$ & 0,452 & & 0,426 & \\
\hline $\begin{array}{l}\text { Aumentadas en } 100 \% \text { recaudación de } \\
\text { la adicional }\end{array}$ & 0,446 & & 0,418 & \\
\hline
\end{tabular}

Fuente: Elaboración propia sobre la base de datos de la encuesta Casen (2017). 
Gráfico 7. INGRESOS MONETARIOS EN CASO DE QUE AUMENTEN TRANSFERENCIAS EN UN 33\% DE LA RECAUDACIÓN ADICIONAL EN AMBOS CASOS

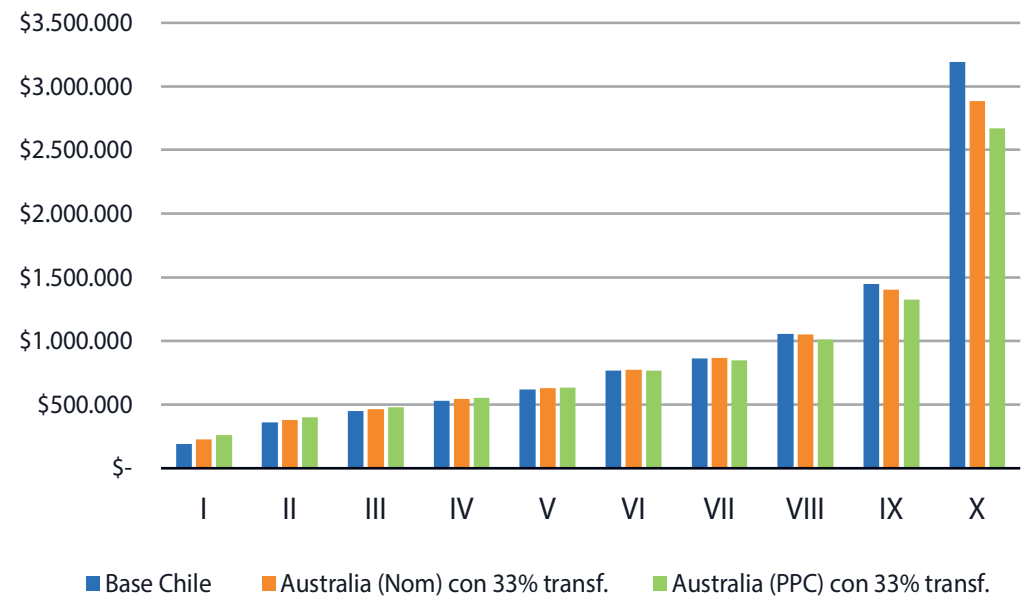

Fuente: Elaboración propia sobre la base de datos de la encuesta Casen (2017).

\section{España}

A continuación replicaremos las simulaciones anteriores para el caso de España. Los valores considerados para los tipos de cambio nominal y PPC serán de $\$ 733,2$ y $\$ 652,8$ por $€ 1$, respectivamente. La Tabla 12 muestra la estructura impositiva para los ingresos anuales de las personas en España.

Como se puede observar, una particularidad de España es que cuenta con impuestos a nivel de gobierno central y a nivel local, en donde hay cinco tramos que tienen tasas que van desde el $9 \%$ hasta el $22,5 \%$. Adicionalmente, no hay tramo exento, pues los ingresos comienzan a pagar inmediatamente una tasa del 9,5\%, tanto por los impuestos centrales como por los locales. Sin embargo, tanto a nivel de gobierno central como del local se cuenta con un descuento personal, ${ }^{41}$ que es una variable dependiente de las características de las personas que viven en el hogar. ${ }^{42}$ Para efectos de este trabajo, consideraremos un descuento

\footnotetext{
${ }^{41}$ Personal Allowance en inglés.

${ }^{42} \mathrm{~A}$ modo de ejemplo, una pareja puede determinar tributar juntos, lo que les permite un descuento de $€ 3.400$. En cambio, un adulto que vive solo con sus hijos tiene derecho a un descuento de $€ 2.150$. Adicionalmente a estos, existen también para familias grandes (más de tres hijos), para trabajadores con discapacidades, para desempleados que aceptan empleos que implican cambios de residencia, entre otros.
} 
personal de $€ 5.550$ anuales, que es el reportado por la OCDE para España, y que asumiremos como representativo, tanto a nivel de gobierno central como de los gobiernos locales.

Tabla 12. ESTRUCTURA IMPOSITIVA PARA LOS INGRESOS DE LAS PERSONAS EN ESPAÑA

\begin{tabular}{ccccc}
\hline \multicolumn{5}{c}{ España - Gobierno central* } \\
\hline$€$ & - & Nivel de ingreso* $^{* *}$ & Tasa (\%) \\
$€$ & 12.450 & $€$ & 12.450 & 9,5 \\
$€$ & 20.200 & $€$ & 20.200 & 12,0 \\
$€$ & 35.200 & $€$ & 35.200 & 15,0 \\
$€$ & 60.000 & $€$ & 60.000 & 18,5 \\
\hline
\end{tabular}

\begin{tabular}{|c|c|c|c|c|}
\hline \multicolumn{5}{|c|}{ España - Gobierno local*, ${ }^{* * *}$} \\
\hline \multicolumn{4}{|c|}{ Nivel de ingreso** } & \multirow{2}{*}{$\begin{array}{c}\text { Tasa }(\%) \\
9,0\end{array}$} \\
\hline$€$ & - & $€$ & 12.450 & \\
\hline$€$ & 12.450 & $€$ & 17.707 & 11,2 \\
\hline$€$ & 17.707 & $€$ & 33.007 & 13,3 \\
\hline$€$ & 33.007 & $€$ & 53.407 & 17,9 \\
\hline$€$ & 53.407 & & más & 21,0 \\
\hline
\end{tabular}

Nota*: Tanto a nivel central como local, se incluye un descuento personal por $€ 5.550$ anuales.

Nota**: En España hay tasas diferenciadas para los ingresos del trabajo y del capital, siendo las máximas tasas del capital de $11 \%$. Para nuestro ejercicio se toman las tasas que paga el capital en Chile.

Nota***: Cada autonomía tiene sus propios tramos. Para efectos de este trabajo consideraremos la comunidad de Madrid, la tercera con mayor población, con un 14\% de la población total en 2018, siendo la que tiene el mayor PIB per cápita del país, con $€ 34.916$ por habitante. ${ }^{43}$ La principal diferencia con otras comunidades es que en algunas de ellas, como en Cataluña y la Comunidad Valenciana, hay tramos sobre los $€ 53.407$, con tasas que llegan hasta el $25,5 \%$.

Fuente: Elaboración propia en base a datos de la OCDE.

La Tabla 13 muestra el ajuste de la estructura tributaria siguiendo el tipo de cambio nominal, donde, adicionalmente, el descuento personal

\footnotetext{
${ }^{43}$ Estos datos fueron extraídos desde el Instituto Nacional de Estadísticas de España. La cifra de población está disponible en https://www.ine.es/jaxiT3/Datos.htm?t=2853\#!tabstabla [7 de julio 2020]. Por su parte, la cifra de PIB per capita está disponible en https:// www.ine.es/prodyser/espa_cifras/2019/31/\#zoom=z [7 de julio 2020].
} 
para ambos niveles equivale a \$4.068.959. Este descuento se aplicará en el pago de IGC.

Tabla 13. Estructura impositiva para los ingresos imponibles anuales de las personas, siguiendo a España por tipo de cambio nominal

\begin{tabular}{lrrrr}
\hline \multicolumn{4}{c}{ Chile / España - Gobierno central (Nominal) } \\
\hline \multicolumn{5}{c}{ Nivel de ingreso } \\
\hline$\$$ & - & $\$ 9.127 .665$ & Tasa (\%) \\
$\$$ & 9.127 .665 & $\$ 14.809 .544$ & 9,5 \\
$\$$ & 14.809 .544 & $\$ 25.806 .731$ & 12,0 \\
$\$$ & 25.806 .731 & $\$ 43.988 .746$ & 15,0 \\
$\$$ & 43.988 .746 & & o más & 18,5 \\
\hline
\end{tabular}

\begin{tabular}{|c|c|c|c|c|}
\hline \multicolumn{5}{|c|}{ Chile / España - Gobierno local (Nominal) } \\
\hline & \multicolumn{3}{|c|}{ Nivel de ingreso } & \multirow{2}{*}{$\begin{array}{c}\text { Tasa (\%) } \\
9,5\end{array}$} \\
\hline$\$$ & - & $\$$ & 9.127 .665 & \\
\hline$\$$ & 9.127 .665 & $\$$ & 12.981 .812 & 11,2 \\
\hline$\$$ & 12.981 .812 & $\$$ & 24.198 .942 & 13,3 \\
\hline$\$$ & 24.198 .942 & $\$$ & 39.155 .116 & 17,9 \\
\hline$\$$ & 39.155 .116 & & o más & 21,0 \\
\hline
\end{tabular}

Fuente: Elaboración propia.

Por su parte, para el tipo de cambio ajustado por PPC, la Tabla 14 refleja la estructura impositiva con la que se gravarán las rentas imponibles anuales totales, donde, adicionalmente, el descuento personal equivale a \$3.623.040. Este descuento se aplicará en el pago de IGC.

Si usamos el tipo de cambio nominal para hacer la equivalencia, se recaudarían US\$16.890 millones por concepto de ISC y se devolverían US\$222 millones por concepto de IGC. Esta devolución se genera principalmente por el descuento personal, ya que hace que las personas tanto a nivel central como local tributen por tramos inferiores a los que lo hicieron por el ISC, lo que hace que el crédito por este concepto sea mayor que el pago que hay que realizar. Esto dejaría a la recaudación por concepto de impuestos a las personas en un total de US\$16.668 millones (ascendiendo a un 6\% del PIB), lo que implica una recaudación adicional 
de US\$13.082 millones anuales o 4,7\% del PIB. Además, esto se traduce en un coeficiente Gini del ingreso monetario de 0,469, siendo 0,017 puntos menor que en el escenario base.

Tabla 14. ESTRUCTURA IMPOSITIVA PARA LOS INGRESOS IMPONIBLES ANUALES DE LAS PERSONAS, SIGUIENDO A ESPAÑA POR TIPO DE CAMBIO AJUSTADO POR PPC

\begin{tabular}{lrrrr}
\hline \multicolumn{4}{c}{ Chile / España - Gobierno central (PPC) } \\
\hline \multicolumn{5}{c}{ Nivel de ingreso } \\
\hline$\$$ & - & $\$ 8.127 .360$ & Tasa (\%) \\
$\$$ & 8.127 .360 & $\$$ & 13.186 .560 & 9,5 \\
$\$$ & 13.186 .560 & $\$ 22.978 .560$ & 12,0 \\
$\$$ & 22.978 .560 & $\$ 39.168 .000$ & 15,0 \\
$\$$ & 39.168 .000 & & o más & 18,5 \\
\hline
\end{tabular}

\begin{tabular}{lrrrr}
\hline \multicolumn{4}{c}{ Chile / España - Gobierno local (PPC) } \\
\hline \multicolumn{5}{c}{ Nivel de ingreso } \\
\hline$\$$ & - & $\$ 8.127 .360$ & Tasa (\%) \\
$\$$ & 8.127 .360 & $\$ 11.559 .130$ & 9,5 \\
$\$$ & 11.559 .130 & $\$ 21.546 .970$ & 11,2 \\
$\$$ & 21.546 .970 & $\$ 34.864 .090$ & 13,3 \\
$\$$ & 34.864 .090 & & o más & 17,9 \\
\hline
\end{tabular}

Fuente: Elaboración propia.

Por su parte, si usamos el tipo de cambio ajustado por PPC, se recaudarían US\$17.153 millones por concepto de ISC y US\$109 millones por concepto de IGC. En este caso, el IGC produce recaudación, ya que el descuento personal es más bajo, generando que el crédito no sea suficiente para suscitar una devolución. Esto dejaría a la recaudación por concepto de impuestos a las personas en un total de US\$17.262 millones (ascendiendo a un 6,2\% del PIB), lo que implica una recaudación adicional de US\$13.675 millones anuales (o 4,9\% del PIB). Además, esto se traduce en un coeficiente Gini de 0,467, siendo 0,019 puntos menor que en el escenario base. 
La Tabla 15 muestra las distintas distribuciones y recaudaciones que se pueden obtener según el grado de transferencias que se hagan. Se observa que en el caso extremo (todo se usa en más transferencias) el Gini podría bajar a 0,42 en ambos casos. Para ilustrar qué sucede con la distribución de los ingresos por decil, en el Gráfico 8 se muestra qué sucede con los ingresos promedio de cada decil cuando el ejercicio se hace con ambos tipos de cambio y se distribuye como transferencia un tercio de la mayor recaudación.

Gráfico 8. INGRESOS MONETARIOS EN CASO DE QUE AUMENTEN TRANSFERENCIAS EN UN 33\% DE LA RECAUDACIÓN ADICIONAL EN AMBOS CASOS

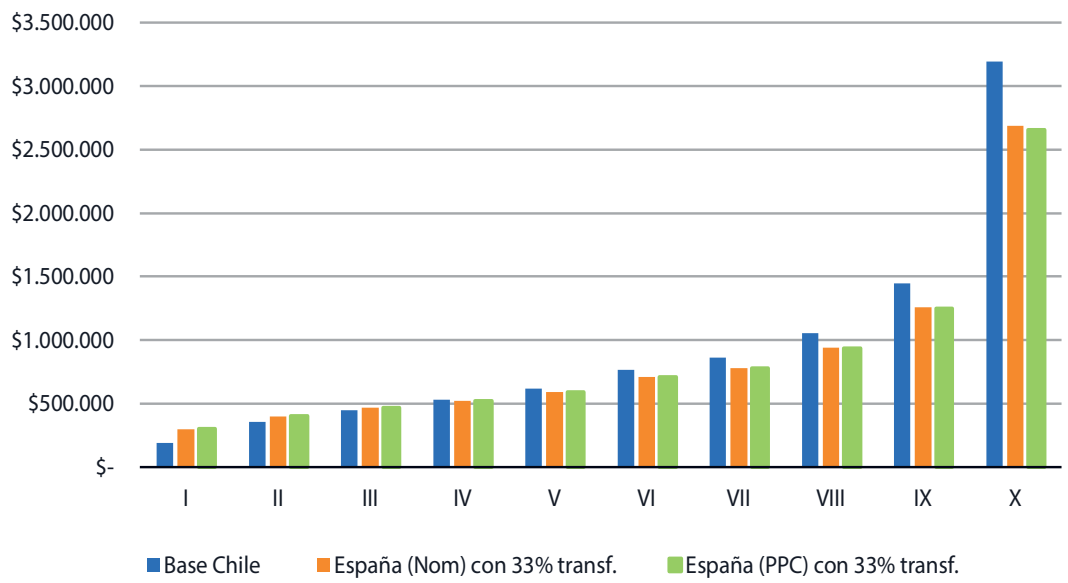

Fuente: Elaboración propia sobre la base de datos de la encuesta Casen (2017).

Es interesante notar que los tramos en España son bastante más cortos que en Australia, lo que hace que una mayor proporción de la población pague mayores tasas. Lo anterior no se corrige incluso con las transferencias. Si se observa el Gráfico 7 (caso australiano), el decil VIII es el primero en mostrar una reducción en el ingreso monetario, mientras que en el caso español el primer decil que ve reducido su ingreso es el IV.

En contraposición, las mayores transferencias generadas por el caso español dejan al decil I con un ingreso de entre $\$ 308$ mil y $\$ 324$ mil (según el tipo de cambio), mientras que en el caso australiano estas magnitudes son de $\$ 226$ mil y $\$ 262$ mil, respectivamente. 
Tabla 15. RESUMEN DE COEFICIENTES DE GINI Y RECAUDACIÓN PARA EL ESTADO DE ACUERDO CON EL AUMENTO DE TRANSFERENCIAS

\begin{tabular}{lcccc}
\hline País & \multicolumn{2}{c}{ Nominal } & \multicolumn{2}{c}{ PPC } \\
\hline España & $\begin{array}{c}\text { Gini } \\
\text { monetario }\end{array}$ & $\begin{array}{c}\text { Recaudación } \\
\text { adicional }\end{array}$ & $\begin{array}{c}\text { Gini } \\
\text { monetario }\end{array}$ & $\begin{array}{c}\text { Recaudación } \\
\text { adicional }\end{array}$ \\
\hline Transferencias base de Chile & 0,469 & 0,467 & \\
$\begin{array}{l}\text { Aumentadas en 33\% de la recaudación } \\
\text { adicional }\end{array}$ & 0,444 & 0,442 & \\
$\begin{array}{l}\text { Aumentadas en 67\% de la recaudación } \\
\text { adicional }\end{array}$ & 0,429 & $\begin{array}{c}\text { USD 13.082 } \\
\text { millones }\end{array}$ & 0,427 & $\begin{array}{c}\text { USD 13.675 } \\
\text { millones }\end{array}$ \\
$\begin{array}{l}\text { Aumentadas en 100\% de la recaudación } \\
\text { adicional }\end{array}$ & 0,421 & & 0.419 & \\
\hline
\end{tabular}

Fuente: Elaboración propia sobre la base de datos de la encuesta Casen (2017).

\section{Noruega}

A continuación replicaremos las simulaciones anteriores para el caso de Noruega. Los valores considerados para los tipos de cambio nominal y PPC serán de $\$ 78,4$ y $\$ 41,5$ por NOK\$1, respectivamente. La Tabla 16 muestra la estructura impositiva para los ingresos anuales de las personas en Noruega.

Tabla 16. ESTRUCTURA IMPOSITIVA PARA LOS INGRESOS DE LAS PERSONAS EN NORUEGA

\begin{tabular}{lrccc}
\hline \multicolumn{5}{c}{ Noruega - Gobierno central } \\
\hline NOK & - & Ingresos de trabajo & Tasa (\%) \\
NOK & 164.100 & NOK & 164.100 & 0,00 \\
NOK & 230.950 & NOK & 230.950 & 0,93 \\
NOK & 580.650 & NOK & 580.650 & 2,41 \\
\hline NOK & 934.050 & NOK & 934.050 & 11,52 \\
\hline
\end{tabular}

\begin{tabular}{lccc}
\hline \multicolumn{4}{c}{ Noruega - Gobiernos central y local* } \\
\hline \multicolumn{4}{c}{ Ingresos totales ${ }^{* *}$} \\
\hline NOK & & o más & Tasa (\%) \\
\hline
\end{tabular}

Nota*: La tasa de $24 \%$ se descompone en un $14,45 \%$ que es destinado a los gobiernos locales, y un $9,55 \%$ que es destinado al gobierno central.

Nota**: Los ingresos totales tienen derecho a dos descuentos mínimos. El primero corresponde a un descuento NOK\$53.150 fijo para todos los contribuyentes. El segundo corresponde a un descuento que corresponde al $44 \%$ de los ingresos del trabajo, con un mínimo de NOK\$31.800 y un máximo de NOK\$94.750.

Fuente: Elaboración propia en base a datos OCDE. 
Como se puede observar, Noruega tiene una estructura similar a la de Chile en cuanto grava los ingresos del trabajo y los ingresos totales de las personas. Sin embargo, a diferencia de Chile, en Noruega el pago de impuestos al trabajo no es crédito para los impuestos totales. Adicionalmente, la tasa a los ingresos totales es fija en $24 \%$, aunque esto se compensa con un descuento personal que va desde los NOK\$84.950 hasta los NOK\$147.900.

La Tabla 17 muestra el ajuste de la estructura tributaria siguiendo el tipo de cambio nominal, donde, adicionalmente, los descuentos mínimos a los ingresos totales van desde $\$ 6.663 .255$ hasta $\$ 11.600 .888$.

Tabla 17. ESTRUCTURA IMPOSITIVA PARA LOS INGRESOS IMPONIBLES ANUALES DE LAS PERSONAS, SIGUIENDO A NORUEGA POR TIPO DE CAMBIO NOMINAL

\begin{tabular}{lrrr}
\hline \multicolumn{4}{c}{ Chile / Noruega - Gobierno central (Nom.) } \\
\hline \multicolumn{4}{c}{ Ingresos del trabajo } \\
\hline$\$$ & - & $\$ 12.871 .574$ & Tasa (\%) \\
$\$ 12.871 .574$ & $\$ 18.115 .113$ & 0,00 \\
$\$ 18.115 .113$ & $\$ 45.544 .664$ & 0,93 \\
$\$ 45.544 .664$ & $\$ 73.264 .434$ & 2,41 \\
$\$ 73.264 .434$ & & o más & 11,52 \\
\hline
\end{tabular}

\begin{tabular}{llcc}
\hline \multicolumn{4}{c}{ Chile / Noruega - Gobiernos central y local (Nom.) } \\
\hline \multicolumn{3}{l}{ Ingresos totales } \\
\hline$\$$ & \multicolumn{2}{c}{ o más } & Tasa (\%) \\
\hline
\end{tabular}

Fuente: Elaboración propia.

Por su parte, para el tipo de cambio ajustado por PPC, la Tabla 18 refleja la estructura impositiva con la que se gravarán las rentas imponibles anuales totales, donde, adicionalmente, los descuentos mínimos a los ingresos totales van desde $\$ 3.521 .153$ hasta $\$ 6.130 .412$.

Es importante notar que para asimilar el sistema de Chile al de Noruega, se hace necesario un ajuste de estructura en el aspecto del cálculo de los impuestos, que consiste en que el ISC imitará las tasas del impuesto al trabajo de Noruega, pero se le agregará un $24 \%$ de tasa en cada tramo. El propósito de agregar este porcentaje es para posteriormente 
permitir que ese $24 \%$ sea crédito para el IGC, de forma de imitar lo que ocurre en Chile.

Tabla 18. ESTRUCTURA IMPOSITIVA PARA LOS INGRESOS IMPONIBLES ANUALES DE LAS PERSONAS, SIGUIENDO A NORUEGA POR TIPO DE CAMBIO AJUSTADO POR PPC

\begin{tabular}{lrrrc}
\hline \multicolumn{4}{c}{ Chile / Noruega - Gobierno central (PPC) } \\
\hline \multicolumn{4}{c}{ Nivel de ingreso } \\
\hline$\$$ & - & $\$ 6.801 .897$ & Tasa (\%) \\
$\$$ & 6.801 .897 & $\$ 9.572 .810$ & 0,00 \\
$\$$ & 9.572 .810 & $\$ 24.067 .773$ & 0,93 \\
$\$ 24.067 .773$ & $\$ 38.716 .099$ & 2,41 \\
$\$ 38.716 .100$ & & o más & 11,52 \\
\hline
\end{tabular}

\begin{tabular}{lccc}
\hline \multicolumn{4}{c}{ Chile / Noruega - Gobiernos central y local (PPC) } \\
\hline \multicolumn{3}{c}{ Nivel de ingreso } \\
\hline$\$ \quad$ o más & Tasa (\%) \\
\hline
\end{tabular}

Fuente: Elaboración propia.

Si usamos el tipo de cambio nominal para hacer la equivalencia, se recaudarían US\$16.945 millones por concepto de ISC y se devolverían US\$1.231 millones por concepto de IGC. Esto dejaría a la recaudación por concepto de impuestos a las personas en un total de US\$15.714 millones (5,7\% del PIB), lo que implica una recaudación adicional de US\$12.128 millones anuales, equivalente a 4,4 puntos del PIB. Además, esto se traduce en un coeficiente Gini del ingreso monetario de 0,482 , siendo 0,004 puntos menor al escenario base.

Por su parte, si usamos el tipo de cambio ajustado por PPC, se recaudarían US\$17.541 millones por concepto de ISC y US\$391 millones por concepto de IGC. Esto dejaría a la recaudación por concepto de impuestos a las personas en un total de US\$17.932 millones (6,5\% del PIB), lo que implica una recaudación adicional de US\$14.345 millones anuales o 5,2\% del PIB. Además, esto se traduce en un coeficiente Gini de 0,476, siendo 0,010 puntos menor que en el escenario base.

La Tabla 19 muestra las distintas distribuciones y recaudaciones que se pueden obtener según el grado de transferencias que se hagan. Se observa que en el caso extremo (todo se usa en más transferencias) el Gini podría bajar a 0,44 y 0,43 en el caso nominal y PPC, respectivamente. 
Para ilustrar qué sucede con la distribución de los ingresos por decil, en el Gráfico 9 se muestra qué sucede con los ingresos promedio de cada decil cuando el ejercicio se hace con ambos tipos de cambio y se distribuye como transferencia un tercio de la mayor recaudación.

Tabla 19. RESUMEN DE COEFICIENTES DE GINI Y RECAUDACIÓN PARA EL ESTADO, DE ACUERDO CON EL AUMENTO DE TRANSFERENCIAS

\begin{tabular}{|c|c|c|c|c|}
\hline \multirow{2}{*}{$\begin{array}{l}\text { País } \\
\text { Noruega }\end{array}$} & \multicolumn{2}{|c|}{ Nominal } & \multicolumn{2}{|c|}{ PPC } \\
\hline & $\begin{array}{l}\text { Gini } \\
\text { monetario }\end{array}$ & $\begin{array}{c}\text { Recaudación } \\
\text { adicional }\end{array}$ & $\begin{array}{l}\text { Gini } \\
\text { monetario }\end{array}$ & $\begin{array}{l}\text { Recaudación } \\
\text { adicional }\end{array}$ \\
\hline Transferencias base de Chile & 0,482 & & 0,476 & \\
\hline $\begin{array}{l}\text { Aumentadas en } 33 \% \text { de la recaudación } \\
\text { adicional }\end{array}$ & 0,459 & & 0,449 & \\
\hline $\begin{array}{l}\text { Aumentadas en } 67 \% \text { de la recaudación } \\
\text { adicional }\end{array}$ & 0,444 & millones & 0,434 & millones \\
\hline $\begin{array}{l}\text { Aumentadas en } 100 \% \text { de la recauda- } \\
\text { ción adicional }\end{array}$ & 0,436 & & 0,427 & \\
\hline
\end{tabular}

Fuente: Elaboración propia sobre la base de datos de la encuesta Casen (2017).

Gráfico 9. INGRESOS MONETARIOS EN CASO DE QUE AUMENTEN LAS TRANSFERENCIAS EN UN $33 \%$ DE LA RECAUDACIÓN ADICIONAL EN AMBOS CASOS

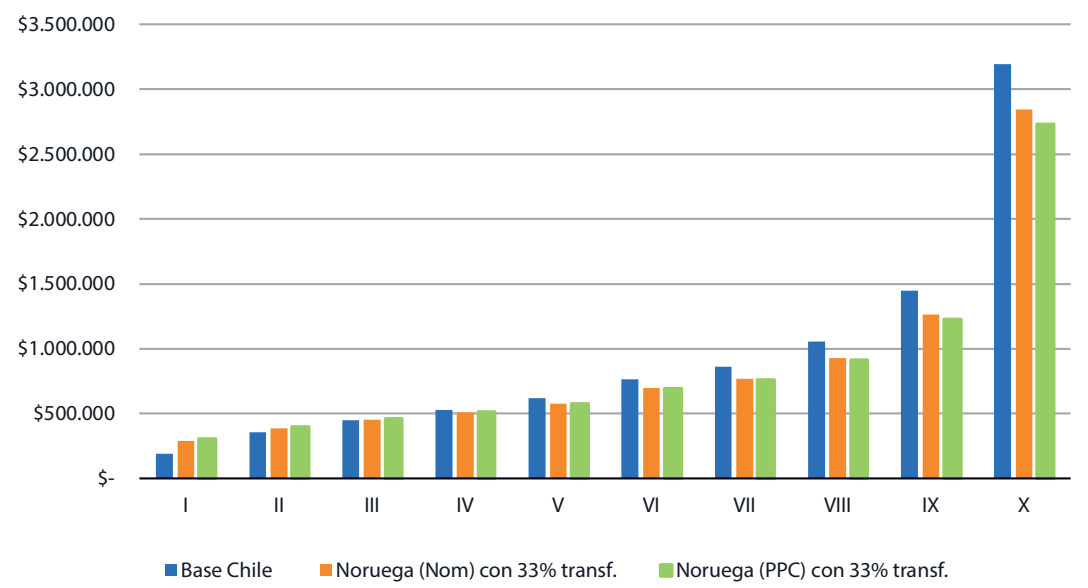

Fuente: Elaboración propia sobre la base de datos de la encuesta Casen (2017). 


\section{Nueva Zelanda}

A continuación replicaremos las simulaciones anteriores para el caso de Nueva Zelanda. Los valores considerados para los tipos de cambio nominal y PPC serán de $\$ 461,15$ y $\$ 283.05$ por NZD\$1, respectivamente. La Tabla 20 muestra la estructura impositiva para los ingresos anuales de las personas en Nueva Zelanda.

Tabla 20. ESTRUCTURA IMPOSITIVA PARA LOS INGRESOS DE LAS PERSONAS EN NUEVA ZELANDA

\begin{tabular}{crccc}
\hline \multicolumn{5}{c}{ Nueva Zelanda - Gobierno central* } \\
\hline \multicolumn{5}{c}{ Nivel de ingreso } \\
\hline NZD & - & NZD & 14.000 & Tasa (\%) \\
NZD & 14.000 & NZD 48.000 & 10,50 \\
NZD & 48.000 & NZD 70.000 & 17,50 \\
NZD & 70.000 & o más & 30,00 \\
\hline
\end{tabular}

Nota*: Además, existe un crédito reembolsable denominado IETC, ${ }^{44}$ que corresponde a NZD 520 para ingresos entre NZD 24.000 y NZD 44.000, y que cae 13 centavos para cada NZD 1 por ingresos mayores a AUD 44.001.

Fuente: Elaboración propia en base a datos de la OCDE.

Como se puede observar, a diferencia de los otros países analizados. Nueva Zelanda tiene solo cuatro tramos. Por otro lado, al igual que Australia, no presenta impuestos locales ni descuentos personales, pero sí presenta alzas importantes de la tasa a medida que los ingresos crecen, y créditos tributarios para los tramos más bajos.

La Tabla 21 muestra el ajuste de la estructura tributaria siguiendo el tipo de cambio nominal, donde, adicionalmente, el IETC beneficia a aquellos con rentas anuales entre $\$ 11.067 .531$ y $\$ 20.290 .473$ con un crédito de $\$ 239.797$. Para aquellos de mayores ingresos, el crédito cae $\$ 13$ por cada $\$ 100$ pesos adicionales de ingreso. Al igual que en el caso de Australia, este crédito será aplicado en el IGC.

\footnotetext{
${ }^{44}$ Independent Earner Tax Credit en inglés. Este crédito tiene otros requisitos adicionales, como que las personas o sus parejas no estén inscritas en determinados programas sociales. Esto genera que la tasa efectiva para los ingresos entre NZD 24.000 y NZD 44.000 sea levemente mayor a la que tienen los ingresos entre NZD 14.000 y NZD 23.999, pero se explica en parte porque este último grupo puede estar recibiendo transferencias. Para efectos de este trabajo asumiremos que todos lo reciben.
} 
Tabla 21. ESTRUCTURA IMPOSITIVA PARA LOS INGRESOS IMPONIBLES ANUALES DE LAS PERSONAS, SIGUIENDO A NUEVA ZELANDA POR TIPO DE CAMBIO NOMINAL

\begin{tabular}{crrrr}
\hline \multicolumn{5}{c}{ Chile / Nueva Zelanda - Gobierno central (Nominal) } \\
\hline \multicolumn{5}{c}{ Nivel ingreso } \\
\hline$\$$ & - & $\$$ & 6.456 .060 & Tasa (\%) \\
$\$$ & 6.456 .060 & $\$$ & 22.135 .062 & 10,5 \\
$\$$ & 22.135 .062 & $\$$ & 32.280 .299 & 17,5 \\
$\$$ & 32.280 .299 & & o más & 30,0 \\
\hline
\end{tabular}

Fuente: Elaboración propia.

Por su parte, para el tipo de cambio ajustado por PPC, la Tabla 22 refleja la estructura impositiva con la que se gravarán las rentas imponibles anuales totales, donde, adicionalmente, el IETC beneficia a aquellos con rentas anuales entre $\$ 6.793 .074$ y $\$ 12.453 .968$ con un crédito de $\$ 147.183$. Para aquellos de mayores ingresos, el crédito cae $\$ 13$ por cada $\$ 100$ pesos adicionales de ingreso.

Tabla 22. ESTRUCTURA IMPOSITIVA PARA LOS INGRESOS IMPONIBLES ANUALES DE LAS PERSONAS, SIGUIENDO A NUEVA ZELANDA POR TIPO DE CAMBIO AJUSTADO POR PPC

\begin{tabular}{lrrrr}
\hline \multicolumn{4}{c}{ Chile / Nueva Zelanda - Gobierno central (PPC) } \\
\hline \multicolumn{5}{c}{ Nivel de ingreso } \\
\hline$\$$ & - & $\$ 3.962 .626$ & Tasa (\%) \\
$\$$ & 3.962 .626 & $\$ 13.586 .147$ & 10,5 \\
$\$$ & 13.586 .147 & $\$ 19.813 .131$ & 17,5 \\
$\$ 19.813 .131$ & & o más & 30,0 \\
\hline
\end{tabular}

Fuente: Elaboración propia.

Si usamos el tipo de cambio nominal para hacer la equivalencia, se recaudarían US\$10.739 millones por concepto de ISC y US\$2.732 miIlones por concepto de IGC. Esto dejaría a la recaudación por concepto de impuestos a las personas en un total de US\$13.470 millones (ascendiendo a un $4,9 \%$ del PIB), lo que implica una recaudación adicional de US\$9.884 millones anuales, equivalente a 3,6\% del PIB. Además, esto se traduce en un coeficiente Gini del ingreso monetario de 0,478 , siendo 0,008 puntos menor al escenario base. 
Por su parte, si usamos el tipo de cambio ajustado por PPC, se recaudarían US\$12.121 millones por concepto de ISC y US\$3.315 millones por concepto de IGC. Esto dejaría a la recaudación por concepto de impuestos a las personas en un total de US\$15.437 millones (5,6\% del PIB), lo que implica una recaudación adicional de US\$11.851 millones anuales, equivalente a $4,3 \%$ del PIB. Además, esto se traduce en un coeficiente Gini de 0,472, siendo 0,014 puntos menor que en el escenario base.

La Tabla 23 muestra las distintas distribuciones y recaudaciones que se pueden obtener según el grado de transferencias que se hagan. Se observa que en el caso extremo (todo se usa en más transferencias) el Gini podría bajar a 0,435 y 0,426 en los casos nominal y PPC, respectivamente. Para ilustrar qué sucede con la distribución de los ingresos por decil, en el Gráfico 10 se muestra qué sucede con los ingresos promedio de cada decil cuando el ejercicio se hace con ambos tipos de cambio y se distribuye como transferencia un tercio de la mayor recaudación.

De forma similar al caso australiano, se genera una recaudación importante, pero menor a los casos español y noruego. Sin embargo, es parecido a los casos anteriormente mencionados en el sentido de que, independientemente del tipo de cambio utilizado, a partir del decil IV los ingresos monetarios se ven reducidos.

Tabla 23. RESUMEN DE COEFICIENTES DE GINI Y RECAUDACIÓN PARA EL ESTADO, DE ACUERDO CON EL AUMENTO DE TRANSFERENCIAS

\begin{tabular}{lcccc}
\hline País & \multicolumn{2}{c}{ Nominal } & \multicolumn{2}{c}{ PPC } \\
\hline Nueva Zelanda & $\begin{array}{c}\text { Gini } \\
\text { monetario }\end{array}$ & $\begin{array}{c}\text { Recaudación } \\
\text { adicional }\end{array}$ & $\begin{array}{c}\text { Gini } \\
\text { monetario }\end{array}$ & $\begin{array}{c}\text { Recaudación } \\
\text { adicional }\end{array}$ \\
\hline Transferencias base de Chile & 0,478 & & 0,472 & \\
$\begin{array}{l}\text { Aumentadas en 33\% de la recaudación } \\
\text { adicional }\end{array}$ & 0,459 & & 0,449 & \\
$\begin{array}{l}\text { Aumentadas en 67\% de la recaudación } \\
\text { adicional }\end{array}$ & 0,445 & $\begin{array}{c}\text { USD 9.884 } \\
\text { millones }\end{array}$ & 0,434 & $\begin{array}{c}\text { USD 11.851 } \\
\text { millones }\end{array}$ \\
$\begin{array}{l}\text { Aumentadas en 100\% de la recaudación } \\
\text { adicional }\end{array}$ & 0,435 & & 0,426 & \\
\hline
\end{tabular}

Fuente: Elaboración propia sobre la base de datos de la encuesta Casen (2017). 
Gráfico 10. INGRESOS MONETARIOS EN CASO DE QUE AUMENTEN TRANSFERENCIAS EN UN 33\% DE LA RECAUDACIÓN ADICIONAL EN AMBOS CASOS

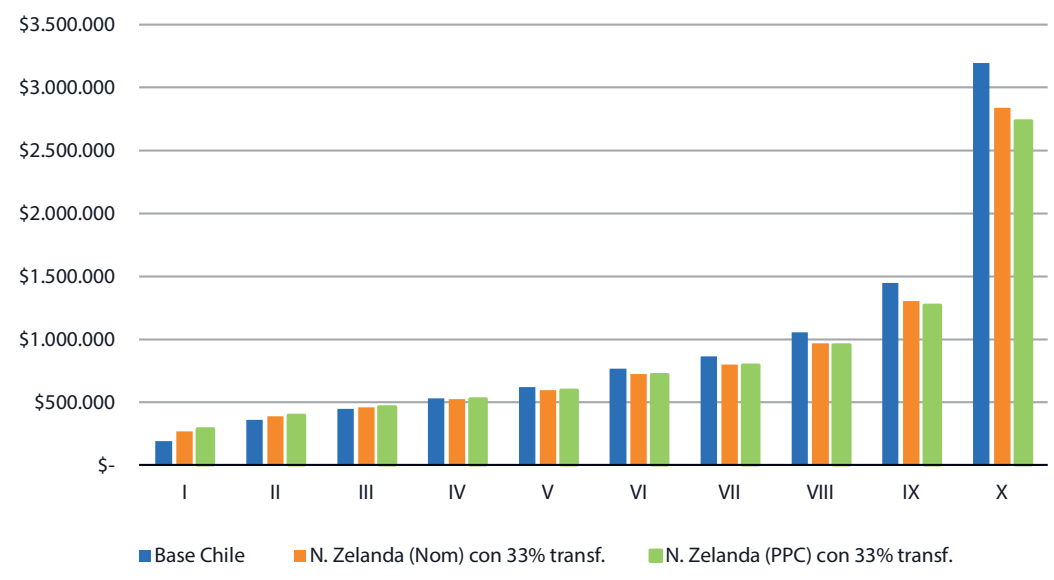

Fuente: Elaboración propia sobre la base de datos de la encuesta Casen (2017).

\section{Conclusiones}

En este artículo aplicamos la estructura al impuesto a la renta personal de cuatro países (Australia, España, Noruega y Nueva Zelanda) a Chile, y medimos cuánto más se recaudaría y cómo variaría la distribución del ingreso. Ello, dado que nuestra estructura de impuesto al ingreso de las personas tiene dos características que la hacen diferente a la de la mayoría de los países del mundo. Primero, el tramo exento es relativamente elevado. Segundo, las tasas marginales suben muy lentamente con el ingreso.

Se concluye, en primer lugar, que de aplicarse la estructura tributaria de estos países a Chile, considerando el tipo de cambio nominal bilateral para los distintos tramos de ingreso, la mayor recaudación fluctuaría entre 1,5\% del PIB para la estructura de Australia hasta 4,7\% del PIB en el caso de España. Si se ajustan las tablas de ingreso al tipo de cambio corregido por PPC, el rango va entre 3\% del PIB para la estructura de Australia hasta 5,2\% del PIB para la de Noruega. En segundo lugar, se concluye que la gran diferencia con Chile no son las tasas marginales máximas, sino las dos características antes mencionadas de nuestro sistema: tramo exento elevado y aumento mucho más gradual de las tasas marginales del impuesto. Tercero, con estas nuevas estructuras de impuesto, la mejora en la distribución del ingreso es marginal. Sin embargo, si estos re- 
cursos extras se usan para aumentar las transferencias, el coeficiente de Gini baja en forma más relevante.

Para ilustrar mejor las diferencias entre la estructura de impuesto a la renta de las personas en Chile en comparación con los países aquí mencionados, la Tabla 24 muestra las tasas medias efectivas de impuesto que se pagan en estos países para distintos niveles de ingreso. Por ejemplo, si consideramos las escalas a tipo de cambio nominal, una persona que en Chile, en 2017, tenía un ingreso imponible de 1 millón de pesos, al mes pagaba una tasa media de $1,5 \%$. Sin embargo, con ese mismo ingreso si se usara la escala de impuestos de esos países pagaría entre $2,8 \%$ y $12,6 \%$. Si el ingreso de una persona es de 10 millones, en Chile aquella pagará $24 \%$, en comparación con un rango de entre $29,5 \%$ y $36,9 \%$ en los otros países. Por cierto, si usamos PPC, las diferencias son aún mayores.

Tabla 24. TASA EFECTIVA DE IMPUESTOS SOBRE INGRESOS BRUTOS DE ACUERDO CON LOS DIFERENTES SISTEMAS

\begin{tabular}{|c|c|c|c|c|c|c|c|c|c|c|}
\hline \multirow{2}{*}{\multicolumn{2}{|c|}{$\begin{array}{l}\text { Ingresos } \\
\text { imponibles }\end{array}$}} & \multirow{2}{*}{$\begin{array}{l}\text { Chile } \\
\text { (\%) }\end{array}$} & \multicolumn{2}{|c|}{ Australia } & \multicolumn{2}{|c|}{ España } & \multicolumn{2}{|c|}{ Noruega } & \multicolumn{2}{|c|}{ Nueva Zelanda } \\
\hline & & & Nominal & PPC & Nominal & PPC & Nominal & PPC & Nominal & PPC \\
\hline$\$$ & 350.000 & 0,0 & 0,0 & 0,0 & 0,6 & 2,6 & 0,0 & 0,9 & 10,5 & 10,9 \\
\hline$\$$ & 500.000 & 0,0 & 0,0 & 0,8 & 6,1 & 7,5 & 0,0 & 4,6 & 10,5 & 10,4 \\
\hline$\$$ & 1.000 .000 & 1,5 & 2,8 & 11,9 & 12,6 & 13,4 & 5,1 & 12,4 & 11,7 & 15,2 \\
\hline$\$$ & 2.000 .000 & 3,9 & 14,4 & 22,6 & 18,9 & 19,9 & 13,2 & 19,4 & 16,6 & 22,3 \\
\hline$\$$ & 5.000 .000 & 13,7 & 26,8 & 32,5 & 30,4 & 31,8 & 23,3 & 30,1 & 26,0 & 28,7 \\
\hline$\$$ & 10.000 .000 & 24,0 & 33,9 & 38,7 & 36,9 & 37,7 & 30,6 & 34,3 & 29,5 & 30,9 \\
\hline
\end{tabular}

Fuente: Elaboración propia.

Si se decidiera ir en la línea de una reforma al impuesto a la renta personal que acercara nuestro sistema al de la mayoría de los países de la $\mathrm{OCDE}$, se podría pensar en uno de los casos o en una combinación de los casos aquí analizados. Además, esto se podría aplicar en forma gradual. Estimamos que, de procederse de esta manera, sería razonable que parte de la mayor recaudación fuera usada para financiar un mayor gasto social y otra parte para reducir el impuesto corporativo. En efecto, la carga tributaria de las empresas en Chile es elevada, bajo los mismos estándares usados en este trabajo. Ello nos hace menos competitivos y daña la inversión y el crecimiento. 


\section{Bibliografía}

Beyer, H. 2011. ¿Que veinte años no es nada...? Una mirada a la desigualdad de ingresos a partir de las encuestas Casen. Estudios Públicos 121, 5-33. Disponible en: https://www.cepchile.cl/cep/site/docs/20160304/20160304095444/rev121_ HBeyer.pdf [20 de julio 2020].

Breeden, C. y Hunter, W. 1985. Tax Revenue and Tax Structure. Public Finance Review 13(2), 216-224.

Briones, I. 2020. Ministro Briones pone como referentes a Australia y Nueva Zelanda para la economía chilena en la próxima década. La Tercera, 31 de diciembre. Disponible en: https://www.latercera.com/pulso/noticia/australia-nuevazelanda-los-referentes-briones-la-economia-chilena/958433/ [2 de enero 2020].

Creedy, J. y Gemmell, N. 2004. The Income Elasticity of Tax Revenue: Estimates for Income and Consumption Taxes in the United Kingdom. The Journal of Applied Public Economics 25, 55-77.

Contreras, D. 1999. Distribución del ingreso en Chile. Nueve hechos y algunos mitos. Perspectivas 2, 311-332.

Djankov, S., Ganser, T., McLiesh, C., Ramalho, R. y Shleifer, A. 2010. The Effect of Corporate Taxes on Investment and Entrepreneurship. American Economic Journal: Macroeconomics 2(3), 31-64.

Encuesta Casen 2017. Resultados encuesta Casen 2017. Disponible en: http:// observatorio.ministeriodesarrollosocial.gob.cl/casen-multidimensional/casen/ casen_2017.php [20 de julio 2020].

Fairfield, T. y Jorrat, M. 2014. Top Income Shares, Business Profits, and Effective Tax Rates in Contemporary Chile. Working Paper 17. International Centre for Tax and Development.

Fuentes, A. y Vergara, R. 2020. Carga tributaria en Chile: evidencia actualizada. Puntos de Referencia 526. Disponible en: https://www.cepchile.cl/cep/site/ docs/20200130/20200130114145/pder526_afuentes_rvergara.pdf [20 de julio 2020].

Hutton, J. y Lambert, P. 1980. Evaluating Income Tax Revenue Elasticities. The Economic Journal 90(360), 901-906.

Jolliffe, D. y Krushelnytskyy, B. 2000. Bootstrap Standard Errors for Indices of Inequality: Inequerr. Stata Technical Bulletin 51.

Kopczuk, W. 2005. Tax Bases, Tax Rates and the Elasticity of Reported Income. Journal of Public Economics 89(11-12), 2093-2119.

Larrañaga, O. y Herrera, R. 2008. Los recientes cambios en la desigualdad y pobreza en Chile. Estudios Públicos 109, 149-186. Disponible en: https://www.cepchile.cl/ cep/site/docs/20160304/20160304094458/r109_larranaga_pobreza.pdf [20 de julio 2020].

Larrañaga, O. y Rodríguez, M. 2014. Desigualdad de ingresos y pobreza en Chile 1990 a 2013. Documento de trabajo. Programa de las Naciones Unidas para el Desarrollo. Chile.

López, R., Figueroa, E. y Gutiérrez, P. 2013. La 'parte del león': nuevas estimaciones de la participación de los súper ricos en el ingreso de Chile. Serie Documentos de Trabajo 379. Departamento de Economía, Universidad de Chile.

López, R., Figueroa, E. y Gutiérrez, P. 2016. Fundamental Accrued Capital Gains and the Measurement of Top Incomes: An Application to Chile. The Journal of Economic Inequality 14, 379-394. 
Rau, T. 2011. Pobreza y desigualdad en Chile: un análisis con la encuesta Casen (163201). En Reinecke, G. y Valenzuela, M.E. (eds.), Chile. El impacto del mercado laboral en el bienestar de las personas. Santiago: Organización Internacional del Trabajo.

Vartia, L. 2008. How do Taxes Affect Investment and Productivity?: An Industry-Level Analysis of OECD Countries. OECD Economics Department Working Papers 656, OECD. EP 